\title{
Foreign Aid Movements in Nepal
}

\author{
Anjay Kumar Mishra*, \& P. S. Aithal**
}

*Post Doctoral Research Scholar, Srinivas University, India and Associate Professor, Madan Bhandari Memorial Academy Nepal, Urlabari-3, Morang, Nepal

OrcidID: 0000-0003-2803-4918; Email: anjaymishra2000@ gmail.com

***Professor, College of Management \& Commerce, Srinivas University, Mangalore, India OrcidID: 0000-0002-4691-8736; E-mail: psaithal@gmail.com

Area/Section: Business Management.

Type of the Paper: Analytical Policy Research.

Type of Review: Peer Reviewed as per $|\mathrm{C}| \mathrm{O}|\mathrm{P}| \mathrm{E} \mid$ guidance.

Indexed in: OpenAIRE.

DOI: https://doi.org/10.5281/zenodo.4677825.

Google Scholar Citation: IJMTS.

\section{How to Cite this Paper:}

Mishra, Anjay Kumar, \& Aithal, P. S., (2021). Foreign Aid Movements in Nepal. International Journal of Management, Technology, and Social Sciences (IJMTS), 6(1), 142-161. DOI: https://doi.org/10.5281/zenodo.4677825.

International Journal of Management, Technology, and Social Sciences (IJMTS)

A Refereed International Journal of Srinivas University, India.

(C) With Author.

CrossRef DOI: https://doi.org/10.47992/IJMTS.2581.6012.0135

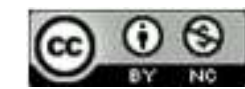

This work is licensed under a Creative Commons Attribution-Non-Commercial 4.0 International License subject to proper citation to the publication source of the work.

Disclaimer: The scholarly papers as reviewed and published by the Srinivas Publications (S.P.), India are the views and opinions of their respective authors and are not the views or opinions of the SP. The SP disclaims of any harm or loss caused due to the published content to any party. 


\title{
Foreign Aid Movements in Nepal
}

\author{
Anjay Kumar Mishra* \& P. S. Aithal** \\ *Post Doctoral Research Scholar, Srinivas University, India and Associate Professor, Madan \\ Bhandari Memorial Academy Nepal, Urlabari-3, Morang, Nepal \\ OrcidID: 0000-0003-2803-4918; Email: anjaymishra2000@ gmail.com \\ **Professor, College of Management \& Commerce, Srinivas University, Mangalore, India \\ OrcidID: 0000-0002-4691-8736; E-mail: psaithal@gmail.com
}

\begin{abstract}
Purpose: Development needs fund and foreign aid is one of the majour source of fund for developing countries. The Paper aims to analyse the trends and composition of foreign aids with case reference to the Swiss aid.

Design/Methodology/Approach: The study is based on secondary data from 2001/02 to 2014/15.Descriptive statistics has been applied to develop the trends and compositions.

Findings/Result: In FY 2014/15, the total foreign aid commitment increased by totaling to Rs.1195.5 million as compared to Rs.2125.9 million in FY 2014/15. Of the total commitment in FY 2014/15 the contribution of bilateral aid was totaling and multilateral aid contributed. While categorizing the total foreign aid the share of grant assistance constituted and loan assistance million. In FY 2014/15 the foreign grant assistant subsequently increased by whereas foreign loan assistance decreased. The bilateral aid disbursement was out of total bilateral aid commitment. Multilateral aid disbursement was 56 percent. The amount of loan is increasing in the economy. This condition also indicates that in future debt burden in budgetary system is directly reducing the development expenditure which decreases the flow of budget in poverty reduction sectors. In amount Swiss aid is much less but is efficiency is widespread in increasing people's living standard. Due to $100 \%$ grant Swiss aid doesn't create fiscal burden in the economy.
\end{abstract}

Originality/Value: it is an empirical research to signify the urgency of increasing Swiss aid and Swiss project in Nepal for sustained and broad-based economic development.

Paper Type: Analytical Policy Research

Keywords: Resource gap, Plans, Expenditure, Disbursement, Commitment

\section{INTRODUCTION :}

Nepal has been receiving aids from many nations. One of the major donors is Switzerland. Because Nepal and Switzerland have geographical similarities, the latter is specially interest in assisting. Switzerland is interest to flow the aid in Nepal and wants to make Nepal as the Switzerland of Asia. Thus, the Swiss aid comes as the relationship between these two countries. Swiss assistance inflow in Nepal is in millions. Assistance from Switzerland is mostly related to the development of the agricultural sector and poverty alleviation, consolidation of democracy and restructuring the civil service of Nepal, which will finally foster the sustainable development of Nepal [1].

Nepal in Asia and Switzerland share some similar characteristics. Both of these countries are mountainous and landlocked and the two peoples have been crossing various geographical and topographical hurdles on the way to economic progress sympathy for Nepalese people and vice versa. The challenges of the lofty Himalayas in Nepal have been accepted by the Swiss and naturally they are interested to provide assistance before the fifties [2].

As a result of this relationship, the Swiss people foresaw the immense possibilities of extending valuable technical assistance to fulfill the needs of economic development here. It is apparent that in Switzerland the private sector is traditionally the forward looking initiator of the industrial and social undertaking. The private sector gets the backing of the government only when the projects become bigger to initiate such government assistance [3]. So, it is important to analyze the trends of Aid in Nepal with a case of 
SWISS which may guide us to improve our economy by overcoming problem of gap in future through our own resources.

\section{OBJRCTIVES :}

The general objective of the study is to analyze the trends and composition of foreign aids with case reference to the Swiss aid.

\section{LITERATURE REVIEW :}

Nepal had begun to receive foreign aid formally after the decline of refine in 1951 from the USA. Since then, the United Sates has been providing technical assistance as well as capital aid in order to help implement projects in virtually every sector of economy. Agriculture, health and transport were the major

areas that have been benefited from the US assistance. In addition, US assistance has also been utilized in various in various institutions building and manpower development activities, India informally provided the aid at that period. A formal agreement concerning economic assistance was not signed until 1954. Before this agreement India had already started assisting Nepal in transport. After the agreement India had already started assisting Nepal in Transport. After the agreement India has also helped Nepal to virtually every field of economic and social development. Some of major areas where Indian aid has been utilized are transportation, irrigation, hydropower, agriculture, horticulture and telecommunication. Canadian aid has been provided as grants and core area of assistance are natural resources management, civil aviation, health and rural development. Like Canadian aid Swiss aid is also devoted to upliftment of the life style of the people who live in remote areas. The multilateral aid in Nepal has begun from 1969. Multilateral agencies to provide aid to Nepal are UNDP, ADB, UNICEF, EC, WB, IDA, UNFPA, WEP etc. Nowadays multilateral aid is increasing more than bilateral aid. On the basis of commitment of aid in fiscal year 2008/09 is sources in out of the total aid Rs. 14426.5 million and bilateral sources cover Rs.9230.8 million (Various issues of Economic Survey, MOF/NG. Nepal [4], [5], [6] and [7].

\section{RESEARCH METHODOLOGY:}

The data have been collected from the publications of finance Minstar, Embassy of Switzerland, Central Bureau of Statistics World Bank, Swiss Agency for development and Co-operation (SDC), UNDP, NRB as secondary sources. The study period is taken from fiscal year 2001/02 to 2014/15 based on availability of data. For the analysis of data quantitative as well as qualitative methods are used. However quantitative techniques have been employed widely. Tabulation of data, trend analysis, graphical presentation of data are applied to make information visible as well as to make them understandable.

\section{RESULTS AND DISCUSSION :}

\subsection{Resource Gap Problem and Need of Foreign aid}

The following table reflects the serious position and financial crisis in Nepal. Each year it has been going in a negative way.

Table 1: Resource Gap in Nepalese Economy

(Rs. in Million)

\begin{tabular}{|l|l|l|l|}
\hline Fiscal Year & Total expenditure & Total revenue & Resource gap \\
\hline $2001 / 02$ & 56118.3 & 32937.9 & 23180.4 \\
\hline $2002 / 03$ & 59579 & 37251 & 22328 \\
\hline $2003 / 04$ & 66272.5 & 42893.8 & 23378.7 \\
\hline $2004 / 05$ & 79835.1 & 48893.6 & 30941.5 \\
\hline $2005 / 06$ & 80072.2 & 50445.5 & 29626.7 \\
\hline $2006 / 07$ & 84006.1 & 56229.8 & 27776.3 \\
\hline
\end{tabular}




\begin{tabular}{|l|l|l|l|}
\hline $2007 / 08$ & 89442.6 & 62331 & 27111.6 \\
\hline $2008 / 09$ & 102560.4 & 70122.7 & 32437.7 \\
\hline $2009 / 10$ & 25761.71 & 22781.2 & 2980.51 \\
\hline $2010 / 11$ & 29536.3 & 24571.1 & 4965.2 \\
\hline $2011 / 12$ & 33916.7 & 28798.4 & 5118.3 \\
\hline $2012 / 13$ & 35863.8 & 33392.7 & 2471.1 \\
\hline $2013 / 14$ & 43505 & 39688 & 3817 \\
\hline $2014 / 15$ & 61710 & 51482 & 10228 \\
\hline Total & $\mathbf{8 9 8 9 0 3 . 4}$ & $\mathbf{6 3 2 1 9 2 . 2}$ & $\mathbf{2 6 6 7 1 1 . 2}$ \\
\hline
\end{tabular}

Source: Various issues of economic survey. MOF/NG Nepal [4] and [5].

To fulfill the development plan outlay and to narrow the resource gap the role of foreign aid is considered to be significant instrument but the resource gap is greater that the foreign aid in each year up to the FY 2014/15. The resources gap was ups and down during the study period. The resource gap is increasing at slow from FY 2001/02 to FY 2004/05. It was fall down and became minimum FY 2010. Again, gap started to rise up to FY 2014/015

\subsection{Foreign aid as Development Expense of Different Plans in Nepal}

Plan is strategies or polices of predetermined courses of action. It is known that main goal of government and fiscal policy are; full employment of resources, economic growth and development, price stability as well as financial sector stability. Since early 1950s the development expenditure and the contribution of the foreign aid in public sector of the different plan period are shown with the help of following table.

Table 2: Share of Foreign Aid and Development Expenses in different Plans

\begin{tabular}{|c|c|c|c|c|c|}
\hline \multirow[t]{2}{*}{ Fiscal Year } & Development & Foreign Aid & $\begin{array}{l}\text { Foreign } \\
\text { Aid }\end{array}$ & \multirow[t]{2}{*}{ Difference } & \multirow[t]{2}{*}{$\begin{array}{l}\text { \% } \\
\text { DE }\end{array}$} \\
\hline & Expenditure & Proposed & Actual & & \\
\hline $1^{\text {st }}$ Plan (1956-61) & 382.9 & 382.9 & 382.9 & 0 & 100 \\
\hline $2^{\text {nd }}$ Plan $(1962-65)$ & 614.7 & 478.3 & 453.6 & -24.7 & 77.8 \\
\hline $3^{\text {rd }}$ Plan $(1965-70)$ & 1639.1 & 919.8 & 1038.5 & 118.7 & 56.1 \\
\hline $4^{\text {th }}$ Plan (1970-75) & 3356.9 & 1509.1 & 1496 & -13.1 & 45.0 \\
\hline $5^{\text {th }}$ Plan (1975-80) & 7545 & 4264.1 & 4182.8 & -81.3 & 56.5 \\
\hline $6^{\text {th }}$ Plan (1980-85) & 21.750 & 13260 & 7924.8 & -5335.2 & 61.0 \\
\hline $7^{\text {th }}$ Plan (1985-90) & 51410 & 23978.5 & 23997.5 & 19 & 46.64 \\
\hline $8^{\text {th }}$ Plan (1992-97) & 113479 & 74355 & 63358.4 & -10996.6 & 65.52 \\
\hline $9^{\text {th }}$ Plan (1997-02) & 189580 & 111546 & 72420 & -39126 & 58.84 \\
\hline $10^{\text {th }}$ Plan (2002-07) & 16200.25 & 11969 & 9131.25 & 2837.75 & 76.29 \\
\hline $11^{\text {th }}$ Plan (2007-09) & 23546.70 & 8413.7 & 6876.07 & 1537.63 & 81.82 \\
\hline $12^{\text {th }}$ Plan $(2009-12)$ & 12958.73 & 24982.00 & 12540.19 & 12441.81 & 50.31 \\
\hline $13^{\text {th }}$ Plan $(2012-15)$ & 32997.6 & 341.59 & 18945.5 & -18604.5 & 25.3 \\
\hline Total & 453732.63 & 276399.99 & 222747.51 & -57227 & 801.12 \\
\hline
\end{tabular}

Source: Various development plan documents. NPC Library/NG, economy Survey MOF/NG and Poverty Reduction Strategy Paper NG/NPC [4] and [5].

The contribution of foreign assistance as the percent of development expenditure had gradually declined from $100 \%$ in the first plan to $45 \%$ in the plan. In the fifth plan it was $56.5 \%$ while in the sixth plan it increased to $61 \%$. No specific improvement in the utilization of foreign aid seemed to have been brought 
in the sixth plan period. In the seventh plan the percent of foreign aid out of the development expenditure was $46.64 \%$. In the eighth plans it increased as $65.5 \%$ but in the ninth plan it had decreased as $58.84 \%$. During the planning of tenth plan period total foreign assistance was estimated differently. This was because government of Nepal and Maoist rebels were negotiating for establishment of peace in Nepal. The total foreign aid is estimated to be Rs. 11969 million. In the eleventh plan period, the contributing of foreign assistance as the percent D.E. has increased to $81.72 \%$ and decreased in $12^{\text {th }}$ plan per and to $50.31 \%$ and $25.3 \%$ in $13^{\text {th }}$ years plan period. The total development expenditure is estimated as Rs.453732.63 million during the first plan to $13^{\text {th }}$ plan of total share of foreign aid development expenditure.

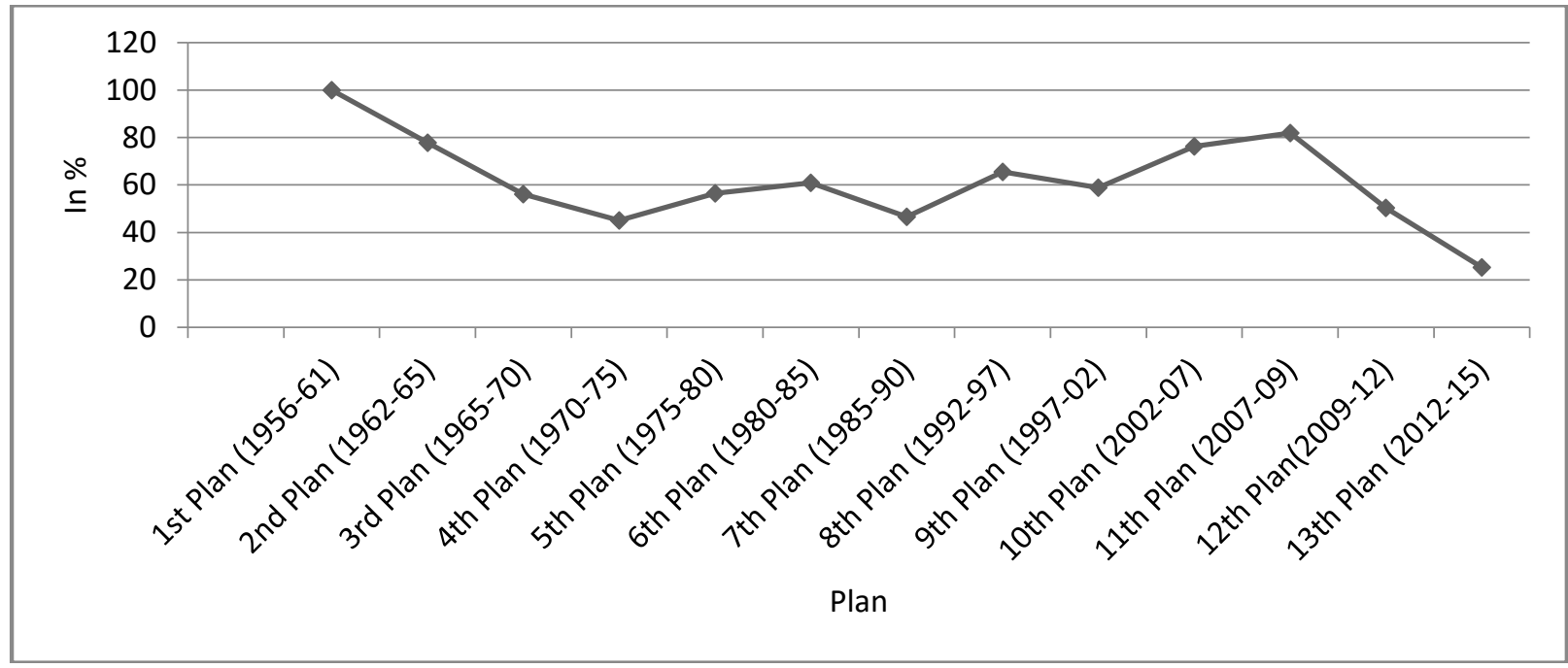

Fig. 1: Foreign Aid as Percent of Development Expenditure in Different Plan of Nepal

Table 5.2 show the actual foreign aid financed in different plan periods. In the first five year plan 100 percent purposed aid was financed. But at the second plan period only 90 percent purposed aid was financed. During the third plan period more than purposed amount of aid was financed. The surplus amount at the period was Rs. 118.7 million i.e., 113 percent achievement observed at the period. At the fourth plan period 99 percent purposed aid was financed and the trend of finance was drastically decreased at fifth five year plan. At that period only 60 percent of purposed aid was financed. In the seventh plan period 100 percent achievement was secured and the ratio was declined as 85 percent in eighth plan period. In the $9^{\text {th }}$ plan period, the purposed ratio was increased as compared to the $8^{\text {th }}$ plan period but the financed ratio declined during the ninth plan period became 64.9 percent. The financed ratio declined during the ninth plan period because of existing internal war in the country. Maoist insurgency increased the uncertainty of the projects and the ratio was declined. Now the $13^{\text {th }}$ plan is running and up to $2014 / 15$ the foreign aid finance is Rs.222747.51 million. In the $11^{\text {th }}$ plan financial ratio inward up to 81.82 percent and 50pervent and 25.3 percent $12^{\text {th }}$ and $13^{\text {th }}$ plan respectively.

The above table and figure clearly show that more than $50 \%$ of development expenditure is directly invested by foreign loan and grants. It means we are deeply dependent on foreign aid to develop our nation. In such condition, the foreign aid plays the major role of development activities.

\subsection{Foreign Aid Commitment and Disbursement}

The existing state of government finance clearly reflects its gradual weak condition over the years due to insufficient mobilization of internal resources. This situation has a rapid growth in debt servicing obligations commensurate with the increase in foreign aid. The obligation of the payments of foreign debts and interest exceeded the rate of gross revenue earnings. Although there was substantial increase in foreign aid commitments due to a low disbursement ratio, there was a big increase in non-disbursed amounts as of not maintaining schedule. 
The delay in the disbursement of the committed foreign aid brings miss-utilization of aid in one hand and adversely effect in the economy by increasing debt burden in the economy. The following table demonstrates the statue of foreign aid commitment and disbursement.

Table 3: Foreign Aid Commitment and Disbursement

\begin{tabular}{|c|c|c|c|}
\hline Fiscal Year & Aid Commitment & Aid Disbursement & $\begin{array}{l}\text { Aid Disbursement as \% } \\
\text { Commitment }\end{array}$ \\
\hline $2001 / 02$ & 32022.1 & 16457.1 & 51.39 \\
\hline $2002 / 03$ & 18352.5 & 16189 & 88.21 \\
\hline $2003 / 04$ & 20448 & 17523.9 & 85.70 \\
\hline $2004 / 05$ & 31287 & 18797.4 & 60.08 \\
\hline $2005 / 06$ & 33227.7 & 14384.8 & 43.29 \\
\hline $2006 / 07$ & 43202.7 & 15885.5 & 36.77 \\
\hline $2007 / 08$ & 23738 & 18912.4 & 79.67 \\
\hline $2008 / 09$ & 38152.3 & 23657.3 & 62.01 \\
\hline $2009 / 10$ & 18846.62 & 12369.9 & 65.63 \\
\hline $2010 / 11$ & 1153.4 & 1079.71 & 93.61 \\
\hline $2011 / 12$ & 7582.79 & 1045.30 & 13.7 \\
\hline $2012 / 13$ & 5507.3 & 9599.5 & 17.4 \\
\hline $2013 / 14$ & 2125.98 & 1036.6 & 48.7 \\
\hline $2014 / 15$ & 1195.56 & 1020.7 & 8.53 \\
\hline Total & 276842 & 167959.1 & 754.69 \\
\hline
\end{tabular}

Sources: Various issues of economic survey MOF/NG [4] and [5].

The above table shows the fluctuating situation of foreign aid commitment and disbursement. In the fiscal year 1992/93 only $54.4 \%$ of total committed aid was disbursed. In fiscal year 1993/94 only $4.72 \%$ of total committed aid was disbursed.

The optimum percentage of the committed foreign aid was disbursed in the fiscal year 1994/95 which was $102.7 \%$. The lowest disbursement rate was in fiscal year 2006/07 which was only $36.7 \%$. In fiscal year 2008/09 $62 \%$ of the committed aid was disburse.

The raising difference between foreign aid commitment and disbursement is necessary to be maintained through continuous follow up and performance as of agreement.

Some objects often show the negative impact in the society and even stop to provide the aid in such a case.

\subsection{Sectoral commitment and Disbursement of Foreign Aid}

During the study period 2001/2002 to 2014/15 total commitment of foreign aid including bilateral and multilateral is Rs. 571790.1 million. On an average the social service sector has given the topmost priority. In this sector Rs. 118438 million aids have been committed by the donors. The second priority has been given to the transport power and communication sector. In this sector Rs.102529.4 million has been committed which is $25.8 \%$ of the total committed amount. The third priority has been given on other sector. In this Rs. 88054.5 million has been committed which is $22.5 \%$ of the total aid. In agriculture, irrigation and forestry sector Rs. 60939.1 million has been committed during given on industry and commerce. In this sector only Rs. 4449.1 million has been committed which is only $1.1 \%$ of the total commitment.

The sectoral distribution of foreign aid shows the amount of aid disbursed in different sectors and identified the sector that has been given priorities. The researcher already identified that during the period of 2001/02 to 2008/09 the topmost propriety of aid commitment is in social services sector. Now the researcher goes to identify the topmost priority of aid disbursement in the selected study period. In 1956 when Nepal entered in planned development, she did not have any infrastructure, transportation and communication. Hilly region was almost exclusively meager in condition. There was urgent need to ling hill and terai sector in development. To fulfill this condition modern transport system, road construction emerged as the priority of investment until 1992/93. 
Table 4: Foreign Aid Commitment and Disbarment by Sectors

\begin{tabular}{|c|c|c|c|c|c|c|c|c|c|c|c|c|c|c|c|c|c|c|}
\hline \multirow{2}{*}{$\begin{array}{c}\text { Fisca } \\
1 \\
\text { Year }\end{array}$} & \multicolumn{3}{|c|}{ Agriculture } & \multicolumn{3}{|c|}{ Transport } & \multicolumn{3}{|c|}{ Industry } & \multicolumn{3}{|c|}{ S. Service } & \multicolumn{3}{|c|}{ Other } & \multirow{2}{*}{$\begin{array}{c}\text { Total } \\
\begin{array}{c}\text { Com } \\
\text { mit }\end{array}\end{array}$} & \multirow[b]{2}{*}{$\begin{array}{c}\text { Disb } \\
\text { ur }\end{array}$} & \multirow[b]{2}{*}{$\%$} \\
\hline & $\begin{array}{c}\text { Com } \\
\text { mit }\end{array}$ & $\begin{array}{c}\text { Disb } \\
\text { ur }\end{array}$ & $\%$ & $\begin{array}{c}\text { Com } \\
\text { mit }\end{array}$ & $\begin{array}{c}\text { Disb } \\
\text { ur }\end{array}$ & $\%$ & $\begin{array}{c}\text { Com } \\
\text { mit }\end{array}$ & $\begin{array}{c}\text { Disb } \\
\text { ur }\end{array}$ & $\%$ & $\begin{array}{c}\text { Com } \\
\text { mit }\end{array}$ & $\begin{array}{c}\text { Disb } \\
\text { ur }\end{array}$ & $\%$ & $\begin{array}{c}\text { Com } \\
\text { mit }\end{array}$ & $\begin{array}{c}\text { Disb } \\
\text { ur }\end{array}$ & $\%$ & & & \\
\hline $\begin{array}{c}1992 / \\
93\end{array}$ & 3901 & $\begin{array}{c}1466 . \\
8\end{array}$ & $\begin{array}{c}37 . \\
6\end{array}$ & 1882 & $\begin{array}{l}312 \\
0.1\end{array}$ & $\begin{array}{c}165 \\
8\end{array}$ & 32.7 & $\begin{array}{c}191 . \\
1\end{array}$ & $\begin{array}{c}584 . \\
4\end{array}$ & $\begin{array}{c}4527 . \\
7\end{array}$ & 845 & 845 & 59.6 & 43.9 & 73.7 & $\begin{array}{c}1040 \\
3\end{array}$ & $\begin{array}{c}5666 . \\
9\end{array}$ & $\begin{array}{c}54.4 \\
7\end{array}$ \\
\hline $\begin{array}{c}1993 / \\
94\end{array}$ & $\begin{array}{c}2992 . \\
4\end{array}$ & $\begin{array}{c}1489 . \\
7\end{array}$ & $\begin{array}{c}49 . \\
8\end{array}$ & $\begin{array}{c}6264 . \\
3\end{array}$ & $\begin{array}{l}267 \\
9.1\end{array}$ & $\begin{array}{c}42.7 \\
7\end{array}$ & 186.4 & $\begin{array}{c}656 . \\
6\end{array}$ & $\begin{array}{c}352 . \\
3\end{array}$ & $\begin{array}{c}3043 . \\
3\end{array}$ & $\begin{array}{c}1555 . \\
8\end{array}$ & 51.1 & $\begin{array}{c}3343 . \\
6\end{array}$ & 64.9 & 1.9 & $\begin{array}{c}1583 \\
0\end{array}$ & $\begin{array}{c}6446 . \\
1\end{array}$ & $\begin{array}{c}40.7 \\
2\end{array}$ \\
\hline $\begin{array}{c}1994 / \\
95\end{array}$ & $\begin{array}{c}3616 . \\
2\end{array}$ & $\begin{array}{c}1253 \\
2\end{array}$ & $\begin{array}{c}34 . \\
7\end{array}$ & 984.2 & $\begin{array}{l}216 \\
4.3\end{array}$ & $\begin{array}{c}219 . \\
9\end{array}$ & 17 & $\begin{array}{c}931 . \\
6\end{array}$ & $\begin{array}{c}548 \\
1\end{array}$ & 954.6 & $\begin{array}{c}1575 . \\
8\end{array}$ & $\begin{array}{c}165 . \\
1\end{array}$ & 93.4 & 65.9 & 70.6 & $\begin{array}{c}5665 . \\
4\end{array}$ & 5991 & $\begin{array}{c}105 . \\
7\end{array}$ \\
\hline $\begin{array}{c}1995 / \\
96\end{array}$ & $\begin{array}{c}5003 . \\
3\end{array}$ & $\begin{array}{c}1945 \\
4\end{array}$ & $\begin{array}{c}38 . \\
9\end{array}$ & $\begin{array}{c}6747 . \\
3\end{array}$ & $\begin{array}{l}248 \\
5.1\end{array}$ & $\begin{array}{c}36.8 \\
3\end{array}$ & 446.4 & $\begin{array}{l}217 \\
4.3\end{array}$ & $\begin{array}{c}487 . \\
1\end{array}$ & $\begin{array}{c}6838 . \\
9\end{array}$ & $\begin{array}{c}1191 . \\
6\end{array}$ & 17.4 & $\begin{array}{c}2048 . \\
2\end{array}$ & 4 & 0.20 & $\begin{array}{c}2108 \\
4.1\end{array}$ & $\begin{array}{c}7800 . \\
4\end{array}$ & $\begin{array}{c}37.0 \\
0\end{array}$ \\
\hline $\begin{array}{c}1996 / \\
97\end{array}$ & $\begin{array}{c}2354 . \\
9\end{array}$ & $\begin{array}{c}1927 . \\
4\end{array}$ & $\begin{array}{c}81 . \\
8\end{array}$ & $\begin{array}{c}5701 . \\
6\end{array}$ & $\begin{array}{l}393 \\
9.6\end{array}$ & 69.1 & $\begin{array}{c}1034 \\
4\end{array}$ & $\begin{array}{c}696 . \\
9\end{array}$ & $\begin{array}{c}67.3 \\
7\end{array}$ & $\begin{array}{c}7323 . \\
5\end{array}$ & $\begin{array}{c}2667 . \\
6\end{array}$ & 36.4 & $\begin{array}{c}4112 . \\
3\end{array}$ & 4.1 & 0.10 & $\begin{array}{c}2052 \\
6.7\end{array}$ & $\begin{array}{c}9235 . \\
6\end{array}$ & $\begin{array}{c}44.9 \\
9\end{array}$ \\
\hline $\begin{array}{c}1997 / \\
98\end{array}$ & $\begin{array}{c}1550 . \\
7\end{array}$ & $\begin{array}{c}5519 . \\
1\end{array}$ & $\begin{array}{c}355 \\
.9\end{array}$ & $\begin{array}{c}5951 . \\
4\end{array}$ & $\begin{array}{l}416 \\
9.4\end{array}$ & $\begin{array}{c}70.0 \\
6\end{array}$ & 46.6 & $\begin{array}{c}389 . \\
9\end{array}$ & $\begin{array}{c}836 . \\
7\end{array}$ & $\begin{array}{c}4674 . \\
2\end{array}$ & $\begin{array}{c}1468 . \\
6\end{array}$ & 31.4 & 949.3 & 10.1 & 1.06 & $\begin{array}{c}1317 \\
2.2\end{array}$ & $\begin{array}{c}1155 \\
7.1\end{array}$ & $\begin{array}{c}87.7 \\
4\end{array}$ \\
\hline $\begin{array}{l}1998 / \\
99\end{array}$ & $\begin{array}{c}3326 . \\
5\end{array}$ & $\begin{array}{c}3462 . \\
4\end{array}$ & $\begin{array}{c}104 \\
.1\end{array}$ & $\begin{array}{c}3152 . \\
3\end{array}$ & $\begin{array}{l}457 \\
4.7\end{array}$ & $\begin{array}{c}145 . \\
1\end{array}$ & 254.1 & $\begin{array}{c}480 . \\
3\end{array}$ & 189 & $\begin{array}{c}1923 . \\
6\end{array}$ & $\begin{array}{c}2680 . \\
1\end{array}$ & $\begin{array}{c}139 . \\
4\end{array}$ & $\begin{array}{c}4220 . \\
1\end{array}$ & 51.7 & 1.2 & $\begin{array}{c}1287 \\
6.6\end{array}$ & $\begin{array}{c}1124 \\
9.2\end{array}$ & $\begin{array}{c}87.3 \\
6\end{array}$ \\
\hline $\begin{array}{c}1999 / \\
00\end{array}$ & $\begin{array}{c}2518 . \\
6\end{array}$ & $\begin{array}{c}3399 . \\
6\end{array}$ & 135 & $\begin{array}{c}8741 . \\
7\end{array}$ & $\begin{array}{l}805 \\
2.7\end{array}$ & $\begin{array}{c}92.1 \\
2\end{array}$ & 0 & 15.9 & - & 4017 & $\begin{array}{c}2636 . \\
2\end{array}$ & 65.6 & $\begin{array}{c}1260 . \\
1\end{array}$ & $\begin{array}{c}84 . \\
6\end{array}$ & 14.6 & $\begin{array}{c}1653 \\
7.4\end{array}$ & $\begin{array}{c}1428 \\
9\end{array}$ & $\begin{array}{c}86.4 \\
0\end{array}$ \\
\hline $\begin{array}{c}2000 / \\
01\end{array}$ & $\begin{array}{c}6589 . \\
5\end{array}$ & $\begin{array}{c}2827 . \\
5\end{array}$ & $\begin{array}{c}43 . \\
6\end{array}$ & $\begin{array}{c}2086 \\
9.3\end{array}$ & $\begin{array}{l}850 \\
4.5\end{array}$ & $\begin{array}{c}40.7 \\
5\end{array}$ & 0 & 23.4 & - & $\begin{array}{c}7618 \\
2\end{array}$ & 3665 & 48.1 & 4666 & 11.5 & 0.25 & $\begin{array}{c}3964 \\
3\end{array}$ & $\begin{array}{c}1503 \\
1.9\end{array}$ & $\begin{array}{c}37.9 \\
2\end{array}$ \\
\hline $\begin{array}{c}2001 / \\
02\end{array}$ & $\begin{array}{c}1299 \\
7.4\end{array}$ & $\begin{array}{c}2852 . \\
1\end{array}$ & $\begin{array}{c}21 . \\
9\end{array}$ & $\begin{array}{c}3806 . \\
7\end{array}$ & $\begin{array}{l}898 \\
9.6\end{array}$ & $\begin{array}{c}236 \\
2\end{array}$ & 156.5 & $\begin{array}{c}198 . \\
1\end{array}$ & $\begin{array}{c}126 . \\
6\end{array}$ & $\begin{array}{c}9993 . \\
2\end{array}$ & $\begin{array}{c}4417 . \\
3\end{array}$ & 66.0 & $\begin{array}{c}8368 . \\
4\end{array}$ & 0 & 0.00 & $\begin{array}{c}3202 \\
2.2\end{array}$ & $\begin{array}{c}1645 \\
7.1\end{array}$ & $\begin{array}{c}51.3 \\
9\end{array}$ \\
\hline $\begin{array}{c}2002 / \\
03\end{array}$ & 3804 & $\begin{array}{c}3383 . \\
9\end{array}$ & $\begin{array}{c}89 . \\
0\end{array}$ & $\begin{array}{c}4696 . \\
9\end{array}$ & $\begin{array}{l}789 \\
2.2\end{array}$ & 168 & $\begin{array}{c}1372 . \\
7\end{array}$ & $\begin{array}{c}404 . \\
7\end{array}$ & $\begin{array}{c}29.4 \\
8\end{array}$ & 3618 & 446.4 & $\begin{array}{c}122 . \\
9\end{array}$ & $\begin{array}{c}4858 . \\
9\end{array}$ & 61.8 & 1.27 & $\begin{array}{c}1835 \\
2.5\end{array}$ & $\begin{array}{c}1618 \\
9\end{array}$ & $\begin{array}{c}88.2 \\
1\end{array}$ \\
\hline $\begin{array}{c}2003 / \\
04\end{array}$ & 685.8 & $\begin{array}{c}3209 . \\
8\end{array}$ & 468 & $\begin{array}{c}9803 . \\
2\end{array}$ & $\begin{array}{l}815 \\
9.3\end{array}$ & $\begin{array}{c}83.2 \\
3\end{array}$ & 876.6 & $\begin{array}{c}298 . \\
5\end{array}$ & $\begin{array}{c}34.0 \\
5\end{array}$ & $\begin{array}{c}6027 . \\
2\end{array}$ & 5794 & 96.1 & $\begin{array}{c}3055 . \\
2\end{array}$ & 62.3 & 2.04 & $\begin{array}{c}2044 \\
8\end{array}$ & $\begin{array}{c}1752 \\
3.9\end{array}$ & $\begin{array}{c}85.7 \\
0\end{array}$ \\
\hline $\begin{array}{c}2004 / \\
05\end{array}$ & $\begin{array}{c}3290 . \\
3\end{array}$ & $\begin{array}{c}3774 . \\
8\end{array}$ & $\begin{array}{c}114 \\
.7\end{array}$ & $\begin{array}{c}2083 . \\
3\end{array}$ & $\begin{array}{c}923 \\
5.1\end{array}$ & $\begin{array}{c}443 . \\
3\end{array}$ & 0 & 19.9 & - & $\begin{array}{c}1936 \\
7.3\end{array}$ & $\begin{array}{c}5759 . \\
5\end{array}$ & 29.7 & 6546 & 8.1 & 0.12 & $\begin{array}{c}3218 \\
6.9\end{array}$ & $\begin{array}{c}1879 \\
7.4\end{array}$ & $\begin{array}{c}60.0 \\
8\end{array}$ \\
\hline $\begin{array}{c}2005 / \\
06\end{array}$ & 748.3 & $\begin{array}{c}3285 . \\
8\end{array}$ & $\begin{array}{c}439 \\
.1\end{array}$ & $\begin{array}{c}6119 . \\
7\end{array}$ & $\begin{array}{l}590 \\
5.7\end{array}$ & 96.5 & 0 & $\begin{array}{c}368 . \\
7\end{array}$ & - & $\begin{array}{c}1525 \\
7.7\end{array}$ & $\begin{array}{c}4685 . \\
8\end{array}$ & 30.7 & $\begin{array}{c}1110 \\
2.2\end{array}$ & $\begin{array}{c}138 . \\
8\end{array}$ & 1.3 & $\begin{array}{c}3322 \\
7.9\end{array}$ & $\begin{array}{c}1438 \\
4.8\end{array}$ & $\begin{array}{c}43.2 \\
9\end{array}$ \\
\hline $\begin{array}{c}2006 / \\
07\end{array}$ & $\begin{array}{c}3976 . \\
1\end{array}$ & $\begin{array}{c}2177 . \\
9\end{array}$ & $\begin{array}{c}54 . \\
8\end{array}$ & $\begin{array}{c}1069 \\
2.3\end{array}$ & $\begin{array}{l}777 \\
4.1\end{array}$ & $\begin{array}{c}72.7 \\
1\end{array}$ & 25.7 & $\begin{array}{c}401 . \\
2\end{array}$ & $\begin{array}{c}156 \\
1\end{array}$ & $\begin{array}{c}1537 \\
0.3\end{array}$ & 5321 & 34.6 & $\begin{array}{c}1313 \\
8.3\end{array}$ & $\begin{array}{c}211 . \\
3\end{array}$ & 1.61 & $\begin{array}{c}4320 \\
2.7\end{array}$ & $\begin{array}{c}1588 \\
5.5\end{array}$ & $\begin{array}{c}36.7 \\
7\end{array}$ \\
\hline $\begin{array}{c}2007 / \\
08\end{array}$ & $\begin{array}{c}2083 . \\
1\end{array}$ & $\begin{array}{c}2429 . \\
6\end{array}$ & $\begin{array}{c}116 \\
.6\end{array}$ & $\begin{array}{c}1910 . \\
7\end{array}$ & $\begin{array}{c}757 \\
0.8\end{array}$ & $\begin{array}{c}396 . \\
2\end{array}$ & 0 & $\begin{array}{c}146 . \\
8\end{array}$ & - & $\begin{array}{c}4568 . \\
3\end{array}$ & $\begin{array}{c}8730 . \\
1\end{array}$ & $\begin{array}{c}191 . \\
1\end{array}$ & $\begin{array}{c}517 \\
6\end{array}$ & 35.1 & 0.23 & $\begin{array}{c}2373 \\
8\end{array}$ & $\begin{array}{c}1891 \\
2.4\end{array}$ & $\begin{array}{c}79.6 \\
7\end{array}$ \\
\hline $\begin{array}{c}2008 / \\
09\end{array}$ & 1601 & $\begin{array}{c}2595 . \\
7\end{array}$ & $\begin{array}{c}162 \\
.1\end{array}$ & $\begin{array}{c}3120 . \\
5\end{array}$ & $\begin{array}{c}986 \\
0\end{array}$ & 316 & 0 & $\begin{array}{c}123 . \\
9\end{array}$ & - & $\begin{array}{c}2837 \\
3.8\end{array}$ & $\begin{array}{c}1074 \\
6.8\end{array}$ & 37.9 & 5057 & $\begin{array}{c}330 . \\
9\end{array}$ & 6.5 & $\begin{array}{c}3815 \\
2.3\end{array}$ & $\begin{array}{c}2365 \\
7.3\end{array}$ & $\begin{array}{c}62.0 \\
7\end{array}$ \\
\hline $\begin{array}{c}2009 / \\
10\end{array}$ & $\begin{array}{c}1079 . \\
1\end{array}$ & $\begin{array}{c}1893 . \\
2\end{array}$ & $\begin{array}{c}176 \\
.4\end{array}$ & $\begin{array}{c}2150 . \\
6\end{array}$ & $\begin{array}{c}567 \\
0.1\end{array}$ & $\begin{array}{c}263 \\
65\end{array}$ & $\begin{array}{c}1837 . \\
2\end{array}$ & $\begin{array}{l}101 \\
2.5\end{array}$ & $\begin{array}{c}55.1 \\
1\end{array}$ & $\begin{array}{c}2982 . \\
3\end{array}$ & $\begin{array}{c}2672 . \\
2\end{array}$ & $\begin{array}{c}89.6 \\
0\end{array}$ & 247.1 & $\begin{array}{l}214 \\
2.6\end{array}$ & $\begin{array}{c}867.0 \\
9\end{array}$ & $\begin{array}{c}8296 . \\
3\end{array}$ & $\begin{array}{c}1339 \\
0.6\end{array}$ & $\begin{array}{c}161 . \\
40\end{array}$ \\
\hline $\begin{array}{c}2010 / \\
11\end{array}$ & $\begin{array}{c}1433 . \\
5\end{array}$ & $\begin{array}{c}1594 . \\
2\end{array}$ & $\begin{array}{c}111 \\
.2\end{array}$ & $\begin{array}{c}5748 . \\
2\end{array}$ & $\begin{array}{l}378 \\
2.3\end{array}$ & $\begin{array}{c}65.7 \\
9\end{array}$ & $\begin{array}{c}1379 . \\
3\end{array}$ & $\begin{array}{l}905 \\
1.2\end{array}$ & $\begin{array}{c}656 . \\
21\end{array}$ & $\begin{array}{c}1920 . \\
2\end{array}$ & $\begin{array}{c}2342 . \\
2\end{array}$ & $\begin{array}{c}121 . \\
97\end{array}$ & $\begin{array}{c}2672 . \\
5\end{array}$ & $\begin{array}{l}253 \\
2.1\end{array}$ & 94.74 & $\begin{array}{c}1315 \\
3.7\end{array}$ & $\begin{array}{c}1930 \\
2\end{array}$ & $\begin{array}{c}146 . \\
74\end{array}$ \\
\hline $\begin{array}{c}2011 / \\
12\end{array}$ & 3801 & $\begin{array}{c}1566 . \\
7\end{array}$ & $\begin{array}{c}41 . \\
2\end{array}$ & 6791 & $\begin{array}{l}789 \\
9.2\end{array}$ & $\begin{array}{c}116 . \\
31\end{array}$ & $\begin{array}{c}1241 . \\
5\end{array}$ & $\begin{array}{l}909 \\
1.2\end{array}$ & 1.47 & $\begin{array}{c}1970 . \\
3\end{array}$ & $\begin{array}{c}2014 . \\
7\end{array}$ & $\begin{array}{c}102 . \\
25\end{array}$ & $\begin{array}{c}3261 . \\
3\end{array}$ & $\begin{array}{c}428 \\
1.3\end{array}$ & $\begin{array}{c}131.2 \\
7\end{array}$ & $\begin{array}{c}1706 \\
5.1\end{array}$ & $\begin{array}{c}2485 \\
3.1\end{array}$ & $\begin{array}{c}145 . \\
63\end{array}$ \\
\hline $\begin{array}{c}2012 / \\
13\end{array}$ & $\begin{array}{c}3716 . \\
1\end{array}$ & $\begin{array}{c}1453 . \\
2\end{array}$ & $\begin{array}{c}39 . \\
1\end{array}$ & $\begin{array}{c}1079 . \\
3\end{array}$ & $\begin{array}{l}342 \\
2.2\end{array}$ & $\begin{array}{c}217 . \\
07\end{array}$ & 541.7 & $\begin{array}{c}909 . \\
3\end{array}$ & $\begin{array}{c}167 \\
86\end{array}$ & $\begin{array}{c}2126 . \\
2\end{array}$ & $\begin{array}{c}2271 . \\
3\end{array}$ & $\begin{array}{c}106 . \\
82\end{array}$ & $\begin{array}{c}4126 \\
3\end{array}$ & $\begin{array}{c}427 . \\
9\end{array}$ & 1.03 & $\begin{array}{c}4872 \\
6.3\end{array}$ & $\begin{array}{c}8483 . \\
9\end{array}$ & $\begin{array}{c}17.4 \\
1\end{array}$ \\
\hline $\begin{array}{c}2013 / \\
14\end{array}$ & $\begin{array}{c}2056 . \\
1\end{array}$ & $\begin{array}{c}2599 . \\
7\end{array}$ & $\begin{array}{c}126 \\
.4\end{array}$ & $\begin{array}{c}1810 . \\
8\end{array}$ & $\begin{array}{l}657 \\
0.8\end{array}$ & $\begin{array}{c}362 . \\
86\end{array}$ & 474.2 & $\begin{array}{c}207 \\
1.2\end{array}$ & $\begin{array}{c}436 . \\
77\end{array}$ & $\begin{array}{c}4127 . \\
1\end{array}$ & $\begin{array}{c}8126 . \\
3\end{array}$ & $\begin{array}{c}196 . \\
90\end{array}$ & 143.2 & $\begin{array}{l}167 \\
2.3\end{array}$ & $\begin{array}{c}1167 . \\
81\end{array}$ & $\begin{array}{c}8611 . \\
4\end{array}$ & $\begin{array}{c}2104 \\
0.3\end{array}$ & $\begin{array}{c}244 . \\
33\end{array}$ \\
\hline $\begin{array}{c}2014 / \\
15\end{array}$ & $\begin{array}{c}1299 . \\
5\end{array}$ & $\begin{array}{c}2862 \\
1.1\end{array}$ & $\begin{array}{c}201 \\
.7\end{array}$ & $\begin{array}{c}4120 . \\
5\end{array}$ & $\begin{array}{l}762 \\
6.9\end{array}$ & $\begin{array}{c}185 . \\
09\end{array}$ & 521.6 & $\begin{array}{c}789 . \\
3\end{array}$ & $\begin{array}{c}151 . \\
32\end{array}$ & $\begin{array}{c}2736 . \\
1\end{array}$ & $\begin{array}{c}1980 . \\
7\end{array}$ & $\begin{array}{c}72.3 \\
9\end{array}$ & $\begin{array}{c}4563 . \\
2\end{array}$ & $\begin{array}{c}546 \\
7.3\end{array}$ & $\begin{array}{c}119.8 \\
1\end{array}$ & $\begin{array}{c}1324 \\
0.9\end{array}$ & $\begin{array}{c}4448 \\
5.3\end{array}$ & $\begin{array}{c}355 . \\
96\end{array}$ \\
\hline
\end{tabular}

Source: Various issues of Economic Survey MOF/NG [4] and [5]. 
Justification:

1. Agriculture sector includes irrigation and forestry

2. Transport sector includes power and communication

3. Industry sector includes commerce

From FY 2001/02 to 2002/03 there was very much fluctuation in total aid commitment and disbursement.51.39\% in FY 2001/02 and $88.21 \%$ in 2002/03. Likewise the share nature was followed by all sectors except industry sector. In FY 2001/02 no aid was committed on industry and commerce sector. The shares of other sector disbursement were decreasing.

From FY 2001/02 to 2002/03 there was very much fluctuation in total aid commitment and disbursement. 51.39\% in FY 2002/02 and 88.21\% in 2002/03. Likewise the share of disbursement in relation to commitment was fluctuation in nature. Such nature was followed by all sectors except industry sector. In FY 2001/02 no aid was committed on industry and commerce sector. The shares of other sector disbursement were decreasing.

From FY 2001/02, 2004/05, 2005/06, 2006/07 and 2012/13 the total aid commitments were increasing but the total aid disbursements increased in 2001/02, 2003/04, 2004/05, 2007/08 and decreased in 2002/03, 2005/06, 2006/07, 2009/10 and 2012/13 the year. In FY 2001/02 total aid commitment was Rs. 32022.2 million and disbursement was Rs. 16457.1 million which is $85.7 \%$ of the committed amount. At that year the biggest aid disbursement in relation to commitment was in agriculture sector which was $468 \%$ and in succeeding two years that ratio was $114.7 \%$ and $439.1 \%$. In transport sector the high ratio of disbursement in relation to commitment remains in each three years. The total ad of the industry sector commitment in Y 2003/04 was Rs. 876.6 million but disbursement was Rs. 298.5 million which was $34.05 \%$ of the committed amount. In succeeding two years no aids were committed but disbursement was Rs. 19.9 million and Rs. 368.7 million respectively. In social service sector 96.1 $\%$ aids were disbursed in relation to commitment in this sector and in succeeding two years the ratio of disbursement in relation to commitment was $29.7 \%$ and $30.7 \%$ respectively. In other sectors the ratio lies $0.24 \%$ to $2.4 \%$.

In FY 2006/07 the total aid commitment was Rs. 43202.7 million and that of disbursement was Rs. 15885.5 million which is $36.77 \%$ of the committed amount. In agriculture sector Rs.3976.1 million aids were committed and that of disbursement was Rs. $2177.9 \%$ million which is $54.8 \%$ of the committed amount. In this sector the amount of commitment was increased but the amount of disbursed and that of industry sector was $1561 \%$. In social service sector only $34.6 \%$ of the committed amount was disbursed.

In FY 2007/08 total aid commitment was Rs.23738 million and that of disbursement Rs. 18912.4 million which is $79.67 \%$ of committed amount. In agriculture sector total aid commitment was decreased and became Rs2083.1 million but disbursement was Rs.2429.6 million which increased as compared to the previous year. In transport sector, the committed amount was heavily decreased but disbursement amount slightly decreased. In industry sector no aid was committed but Rs.146.8 million aids were disbursed. In other sectors only $0.23 \%$ of committed aid was disbursed.

In FY 2008/09 the total aid commitment was Rs.23738 million and that of disbursement Rs.18912.4 million which is $79.67 \%$ of committed amount. In agriculture sector total aid commitment was decreased and became Rs.2083.1 million but disbursement was Rs.2429.6 million which increased as compared to the previous year. In transport sector, the committed amount was heavily decreased but disbursement amount slightly decreased. In industry sector no aid was committed but Rs.146.8 million aids were disbursed. In other sectors only $0.23 \%$ of committed aid was disbursed.

In FY 2008/09 the total aid commitment increased by Rs.14414 million and became Rs.38152.3 million. The aid disbursement also increased by Rs.4744 million and became Rs.23657.3 million which is $62.01 \%$ of the committed amount. In transport sector Rs.3120.5million aid is committed but disbursement is Rs.9860 million which is $316 \%$ of the committed amount. Both committed and disbursed amount increased as compared to the previous years. In industry sector no aid is committed but Rs.123.9 million aids were disbursed. In social service sector Rs.28377.8 million aids were committed amount. In other sectors Rs.5057.00 million aids were committed but only Rs.330.9 million aids were disbursed. 
In FY 2009/10 the total and commitment was Rs. 8296.3 million and that disbursement Rs.13390.6 which Rs. 161.40 of committed amount. In annual sector total aid commitment was Rs.350424.2 million decreased as companied to the premise year. In transport sector the total aid commitment Rs.59813 million in social services sector Rs. 118438 million.

In FY 2010/11 the total aid commitment was Rs.13153.7 million and total aid disbursement Rs. 19302 million which is $146.74 \%$ of committed amount. In agriculture, transport, industry and in and service sector the total aid disbursement was Rs.1594.2 million, 3782.3 million, 9051 million 2342 million respectively FY. The total aid commitment in agriculture, transportation, industry and in social sector are Rs.1434, Rs.5748, Rs.1379 and Rs.1920.2 million respectively.

In FY 2011/12 the total aid commitment was Rs. 17065.1 million and that of disbursement Rs. 24853.1 million which Rs. $145.63 \%$ of committed amount. In Agriculture, Transport, Industry and in service sector total aid commitment was Rs.3801, Rs. 6791, Rs. 1242, Rs. 1970.3 million and total disbursement was Rs. 1566.7, Rs. 7899.2, Rs. 9091, Rs. 2015 million respectively. In FY 2012/13 total aid commitment was Rs 48726.3 million and disbursement was Rs 8483.9million. In Agriculture, Transport, Industry and in service sector total aid commitment was Rs. 3716, Rs. 1079, Rs. 541.7, Rs. 2126.2 million respectively and total disbursement was Rs. 1453.2, Rs. 3422.2, Rs. 909.3, Rs. 2271million respectively. FY 2013/14 total aid commitment Iwas Rs. 8611.4 million and disbursement was Rs. 21040.3 million. In Agriculture, Transport, and Industry and in service sector total aid commitment was Rs 2056, Rs 1811, and Rs 474.2, Rs. 4127.1 million respectively. Total aid disbursement was Rs. 2599.7, Rs.6570.8, Rs. 2071, Rs. 8126 million respectively. FY 2014/15 total aid commitment is Rs 13204.9 million and total disbursement Rs 4448.3 million. In Agriculture, Transport, and Industry and in service sector total aid commitment was Rs.1300, Rs. 4121, Rs. 521.6, Rs. 2736.1 and disbursement was Rs. 2861, Rs 7626.9, Rs. 789.3, Rs. 1981 million respectively.

In total Rs.60939.1 million aids are committed in agriculture sector and that of disbursement is Rs. 47000.7 million which is $77.1 \%$ of the committed amount. In transport power and communication sector Rs.102529.4 million aids are committed and that of disbursement is Rs.105076.3 million which is $102.5 \%$ of the committed amount. In industry and commerce sector Rs.4449.1 million aids have been committed and Rs.7522 million is disbursed which is $169.1 \%$ of the committed amount this sector. In social service sector only $48.6 \%$ of the committed aid has disbursed and that is $1.46 \%$ in other sectors. The table 4 exhibits the fluctuation condition in aid commitment and disbursement during the study period. In sector wise analysis there exits non-related condition between aid commitment and disbursement i.e., both commitment and disbursement heavily increased in one year and the decrease in the other. Transport sector was mostly given the topmost priority in both aid commitment and disbursement in each year except FY 2007/08 and 2012/13. It clarifies that donors are more interested to build infrastructures than sectoral development and poverty reduction of country.

\subsection{Total Aid Commitment and Share or Bilateral Aid}

During the study period 2001/02 to 2014/15, the total foreign aid commitment is Rs.571790 million and the share of bilateral aid is $36.67 \%$, which is Rs.209659 million. The share of multilateral aid is $63.3 \%$ less than bilateral aid commitment. In the share bilateral aid $80.36 \%$ amount consists grants and only $19.63 \%$ of committed amount consists loans. The grant is Rs.154257.3 and loan is only Rs.30293.02 million. Table 5 shows the trend of bilateral aid during the study period.

Table 5 : Total Aid Commitment and the share Bilateral Aid

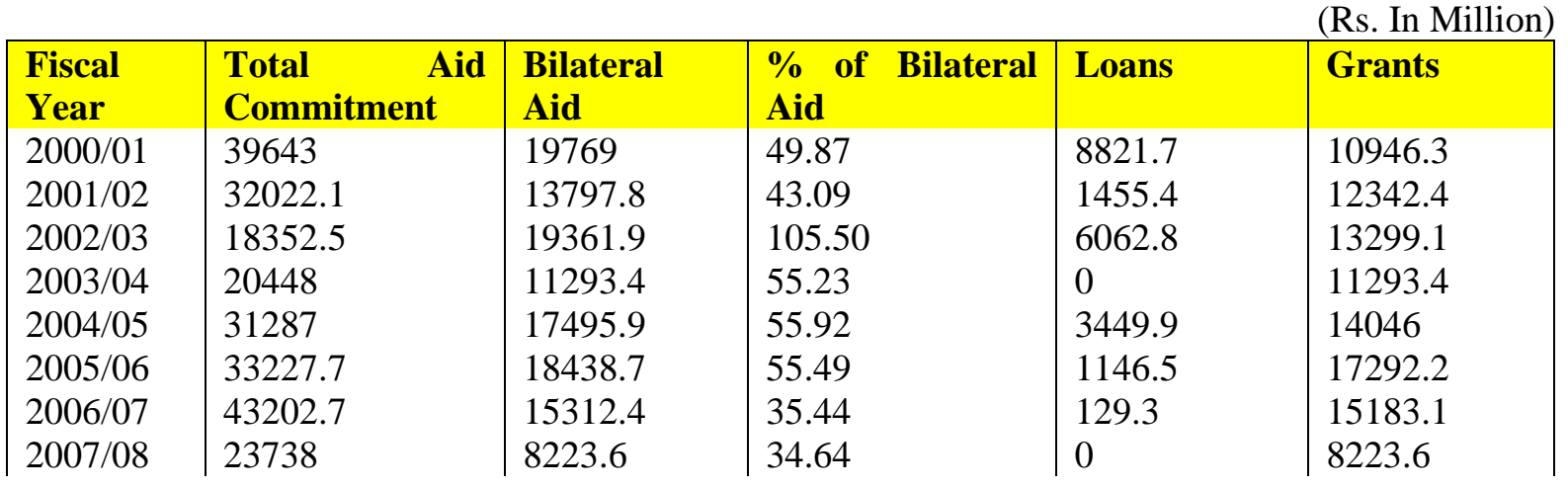




\begin{tabular}{|l|l|l|l|l|l|}
$2008 / 09$ & 38152.3 & 21225.6 & 55.63 & 0 & 21225.4 \\
$2009 / 10$ & 18846.6 & 4109.5 & 21.80 & 1079.54 & 2158.7 \\
$2010 / 11$ & 11534 & 6216.6 & 53.89 & 1122.34 & 3854.6 \\
$2011 / 12$ & 75827.9 & 59128 & 77.9 & 1207.56 & 4592.22 \\
$2012 / 13$ & 55073 & 35230 & 63.9 & 1108.31 & 4081.03 \\
$2013 / 14$ & 21259.8 & 4138.5 & 194.6 & 1196.90 & 3523 \\
$2014 / 15$ & 11955.6 & 3854.6 & 32.2 & 3512.77 & 12196.25 \\
Total & $\mathbf{4 7 4 5 7 0 . 2}$ & $\mathbf{2 5 7 5 9 5 . 5}$ & $\mathbf{9 3 5 . 1}$ & $\mathbf{3 0 2 9 3 . 0 2}$ & $\mathbf{1 5 4 2 5 7 . 3}$ \\
\hline
\end{tabular}

Source: Various Issue of Economic Survey MOF/NG from www.mof.gov.np [4] and [5].

The table 5 clearly shows that there is so much fluctuation in the total aid commitment during the study period. The total aid commitment in the FY 2001/2002 is Rs. 39643 million and bilateral aid is Rs.19769 million. The percent bilateral aid is 49 percent. The trend of loan is Rs. 8821.7 million and grant is Rs 10946.3 million. The total aid is commitment increased in FY 2006/07, 2011/12, 2012/13 while it is decreased in 2013/14 and 2014/15. The bilateral aid is also increased with aid and decreased with aid. In FY 2013/14 and 2014/15 it is 4138.5 million and 351277 million in year 2014/15. Similarly, the grant is Rs. 3523 million in FY 2013/14 and Rs. 12196.25 million in FY 2014/15.

The maximum amount of grants commitment in bilateral aid shows that donors are really interested to develop our nation. They were increased to build infrastructures in the development of recipient country.

Multilateral Aid found $69.78 \%$ and Bilateral Aid found $30.22 \%$ only which shows the highest percentage of share in bilateral aid. It also indicates that bilateral aid is good for the economy. It creates low burden in budgetary allocation.

Table 6: Bilateral Aid Distribution and the Share of Grant and Loan

\begin{tabular}{|l|l|l|}
\hline Bilateral Aid Disbursement & \% Share & Amount Rs. in Million \\
\hline Grants & 83.4 & 68120.1 \\
\hline Loans & 16.6 & 13599.3 \\
\hline Total & 100 & \\
\hline
\end{tabular}

Table 6 clarifies the condition of the bilateral aid disbursement during the study period. The disbursement of the total aid from FY 2001/2002 is 2008/09 total is increasing in nature. In the year $2008 / 10$ the disbursement of aid is more than other years. Likewise, the disbursement of the bilateral aid also increased FY 2014/15 and the nature is increasing and decreasing by year wise. The plant age of bilateral aid is in one year 2006/07, 2010/11 and 2013/14 and in 2014/15. The total loan Rs. 37817.2 million and grant Rs. 92944.6

The composition of bilateral aid reflects maximum grant which indicates low burden of aid in the economy but the debt burden in the economy. The high percentage of multilateral aid directly effect to the government budget. Increasing multilateral aid shows increasing allocation of loan and interest paid amount in the budget which reduces the development expenditure.

When Nepal entered the structural adjustment program, the share of multilateral loan is increasing. In 2006/07 government restructured the central bank and gave administration contract of two commercial banks for the purpose of restructuring and collecting bad debt. For this purpose, the government interment inverted billion rupees of World Bank's loan but still the two banks are holding high share of bad debts. Increasing bad debts in the bank has been questioned about financial sector reform. Likewise poor performance of structural adjustment program, financial sector reform, private and public sector reform only increase multilateral loans and create debt burden in the economy.

\subsection{Trend and Structure of Swiss Aid in Nepal}

Since 1958, the Swiss aid played a vital role in the economic development of Nepal. The Swiss aid after the restoration of democracy emphasized the natural resources management i.e., agricultural sector, 
transport and communication social service specially community development and empowerment of Nepalese people. The aid inflow by Swiss Agency for Development and Co-operation (SDC) though District Development Committee (DDC) and Village Development Committee (VDC) played vital role to sustain development of democratic institution and sentiments of Nepalese people.

Table 7: Trends and Structure of Swiss aid in Nepal

\begin{tabular}{|l|c|c|}
\hline Fiscal Year & Swiss Aid Inflow (Grants) & Percentage Change \\
$2000 / 01$ & 637.67 & -19.76 \\
$2001 / 02$ & 846.77 & 32.79 \\
$2002 / 03$ & 903.57 & 6.71 \\
$2003 / 04$ & 932.72 & 3.23 \\
$2004 / 05$ & 909 & -2.54 \\
$2005 / 06$ & 1217.13 & 33.90 \\
$2006 / 07$ & 1312.78 & 7.86 \\
$2007 / 08$ & 1287.13 & -1.95 \\
$2008 / 09$ & 1187.18 & -7.77 \\
$2009 / 10$ & 2184.7 & 84.02 \\
$2010 / 11$ & 2763.2 & 26.4 \\
$2011 / 12$ & 3341.7 & 20.93 \\
$2012 / 13$ & 4176.7 & 24.98 \\
$2013 / 14$ & 3385.3 & -23.37 \\
$2014 / 15$ & 3246.7 & -4.49 \\
Total & $\mathbf{2 8 3 3 2 . 2 5}$ & $\mathbf{1 8 0 . 9 4}$ \\
\hline
\end{tabular}

Source: various issue of SDC country program reports, SWC budget and annual reports and Foreign Aid division MOF/NG [5], [9], [10], and [11].

Table 7 show the total Swiss aid inflow during the trendy period. In the trendy the total twice aid inflow is Rs. 397714 million. The table also shows the percentage change in Swiss aid in each individual year. In FY 2001/02 the Swiss aid inflow in Nepalese economy was 846.77 which increase in FY 2004/05, 2007/08, 2008/09 up to FY 2012/13 and decrease in FY2013/14, 2014/15. In this year 10.34\% and decreased as compared to the previous year. In FY 1992/93 the Swiss aid again decreased in comparison to FY 1993/94. The Swiss aid inflow FY 2005/6 is 1217.13 million with 33.90 percent aid increment and similarly in FY 2006/7 with 7.86 percent aid increment and decrease 2007/8, 2008/9. In FY 2009/10 Swiss aid inflow was increased by Rs. 2184.7 million, FY 2010/11 by 2763.3 million and Rs. 3341.7 million in 2011/12, Rs. 4176.7 million FY 20012/13 with percent increased by 84.02, 26.47, 20.93, and 24.98 respectively. In $2013 / 14$ it decreased with the 23.37 percent and 4.9 percent as compared previous year.

The structure of the Swiss aid is almost granted. No amount of the loan has been disbursed in the economy during the study period. It indicates that the Swiss aid creates no debt burden in the economy. Swiss government increases aid when democratic institutions were fully functioning in the nation.

\subsection{Swiss Aid Commitment and Disbursement}

During the study period there is no vast gap between the Swiss aid commitment and disbursement. 94\% percent of the total committed aid is disbursed during the study period. At the beginning of the study period only Rs.127.9 million of the total aid was committed but the disbursement amount was Rs.546.27 million which was $417.10 \%$ of the total aid commitment. Total 8 show the situation of the Swiss aid commitment and disbursement.

Table 8: Swiss Aid Commitment and Disbursement

\begin{tabular}{|l|c|c|c|}
\hline $\begin{array}{l}\text { Fiscal } \\
\text { Year }\end{array}$ & $\begin{array}{c}\text { Total Aid } \\
\text { Commitment }\end{array}$ & $\begin{array}{c}\text { Swiss aid } \\
\text { Disbursement }\end{array}$ & $\begin{array}{c}\text { Risbursement as \% of } \\
\text { Commitment }\end{array}$ \\
$2000 / 01$ & 849.94 & 637.67 & 75.03 \\
$2001 / 02$ & 888.8 & 846.77 & 95.27
\end{tabular}




\begin{tabular}{|l|c|c|c|}
$2002 / 03$ & 996.5 & 903.57 & 90.67 \\
$2003 / 04$ & 996.82 & 932.72 & 93.57 \\
$2004 / 05$ & 971.69 & 909 & 93.55 \\
$2005 / 06$ & 1270.6 & 1217.13 & 95.79 \\
$2006 / 07$ & 1292.35 & 1312.78 & 101.58 \\
$2007 / 08$ & 1399 & 1287.13 & 92.00 \\
$2008 / 09$ & 1290.84 & 1187.18 & 91.97 \\
$2009 / 10$ & 1300.0 & 2185.4 & 16.81 \\
$2010 / 11$ & 3702.2 & 2763.2 & 74.63 \\
$2011 / 12$ & 1010 & 3341.7 & 33.08 \\
$2012 / 13$ & 1080 & 4176.7 & 38.67 \\
$2013 / 14$ & 2450 & 3385.3 & 13.81 \\
$2014 / 15$ & 2500 & 3246.7 & 12.98 \\
Total & $\mathbf{2 1 9 9 8 . 7 4}$ & $\mathbf{2 8 3 3 2 . 9 5}$ & $\mathbf{1 0 1 9 . 4 1}$ \\
\hline
\end{tabular}

Source: Various issues of SDC country program reports, SWC budget and annual reports and Foreign Aid division MOF/NG [4], [5] and [11]

Table8 shows the high rate of disbursement of the Swiss aid in relation to commitment. The higher disbursement rates were in the fiscal years 2009/10, 2010/11, 2011/12 and 2012/13. In these years the disbursement as $\%$ of commitment is $16.81,74.63,33.08$, and 38.67 committed aid was disburse. In FY $2013 / 14$ and 2014/15, the disbursement \% as commitment was 13.81 and $12.96 \%$.

With preferring amount, the largest amount of Swiss aid disbursement was in the FY 2012/13. At that year Rs.4176.7 million aids were disbursed. The second largest amount was disbursed in FY 2013/14 which was Rs. 3385.3 million. The third largest amount of the aid disbursement was in the FY 2001/2002 which was Rs.3341.7 million. The lowest amount was disbursed in FY 2001/2002 which was Rs. 846.7 million.

The committed amount was beginning with Rs. 846.7 million and not exceeding Rs. 2500 million. It is because of weak performance of foreign aid utilization in Nepal. The various reports of SDC clearly indicate the low performance of foreign aid utilization in Nepal. The weak democratic institution and rampant corruption in project management harness of Swiss government and individual to increase the amount of Swiss aid in Nepal. However, the commitment of the aid in relation to the foreign aid in relation to the foreign aid and inflow the performance of the Swiss aid is very strong in relation to other foreign aid. The direct inflow of other aid is through government but the Swiss aid inflow through government, projects handled by Swiss people SDC, NGO, INGO sectors. In totality more than $94 \%$ of the committed aid is disbursed. It means highest amount of aid inflow in relation to commitment. The lowest disbursed ratio in relation to commitment is $72 \%$ in fiscal year 2001/2002 which is Rs. 486.77 million. In 14 years study period, 8 years aid disbursement ratio is more than $80 \%$ and only 6 year aid disbursement ratio is less than $80 \%$. The Swiss aid commitment and disbursement figure clearly indicate high association between aid commitment and disbursement.

\subsection{Total Aid Commitment and Swiss aid Commitment}

Although affordable activities are ongoing in Nepal, the Swiss aid commitment in relation to the total aid commitment in less than $4 \%$ in various years in the study period.

The low performance of the Swiss aid commitment in relation to total relation aid commitment. In the

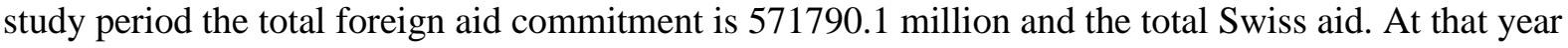
the total Swiss aid commitment was 21998.74 million and the total aid commitment was Rs.28332.95. After 2001/2002 the total aid commitment increased as compared to the previous year but the Swiss aid does not increase as fluctuations in total aid commitment.

Table 9: Total aid commitment and Swiss aid Commitment

(Rs. In Million)

\begin{tabular}{|l|l|l|l|}
\hline Fiscal Year & Total Aid Disbursement & Swiss aid Commitment & \% \\
$2000 / 01$ & 39643 & 849.94 & 2.14 \\
$2001 / 02$ & 32022.1 & 888.8 & 2.78 \\
$2002 / 03$ & 1852.5 & 996.5 & 5.43
\end{tabular}




\begin{tabular}{|l|l|l|l|}
$2003 / 04$ & 20448 & 996.82 & 4.87 \\
$2004 / 05$ & 31287 & 971.69 & 3.11 \\
$2005 / 06$ & 33227.7 & 1270.6 & 3.82 \\
$2006 / 07$ & 43202.7 & 1292.35 & 2.99 \\
$2007 / 08$ & 23738 & 1399 & 5.89 \\
$2008 / 09$ & 38125.3 & 1290.84 & 3.38 \\
$2009 / 10$ & 1884.66 & 1300 & 6.89 \\
$2010 / 11$ & 1153.4 & 370.22 & 32.09 \\
$2011 / 12$ & 7582.79 & 1010 & 1.33 \\
$2012 / 13$ & 5507.3 & 1080 & 1.96 \\
$2013 / 14$ & 2125.91 & 2450 & 1.15 \\
$2014 / 15$ & 1195.56 & 2500 & 2.09 \\
Total & $\mathbf{2 8 2 9 9 5 . 9 2}$ & $\mathbf{1 8 6 6 6 . 7 6}$ & $\mathbf{7 9 . 9 2}$ \\
\hline
\end{tabular}

Source: various issues of Economic Survey MOF/NG, SDC country program reports, SWC budget and annual reports and Foreign Aid division MOF/NG [5] and [12].

The Swiss aid commitment occupied less than 3\% in total aid commitment in fiscal years 2001/02 and 2006/07. Similarly, the Swiss aid commitment was less than $7 \%$ in remaining years except FY 2010/2011. This is the low share or the Swiss aid in foreign aid commitment.

The various issues of SDC country reports clearly indicate effective aid management mechanism of the Swiss government Swiss government and Swiss foreign aid ministry's motto for aid management is to establish projects in rural areas and far remote areas and directly involvement of people for the sharing of benefits. They want to invest least but effectively. Thus, there can't be seen fluctuation in the Swiss aid commitment in relation to the total aid commitment aid disbursement is only $57.82 \%$ of the committed amount. The Swiss aid disbursement is more than $94 \%$ of the committed amount. So, it seems to be greater than the committed amount. Swiss aid disbursement is steady in nature. No more fluctuation in commitment and disbursement.

Table 10: Total Aid Disbursement and Swiss Aid disbursement

\begin{tabular}{|l|c|c|c|}
\hline $\begin{array}{l}\text { Fiscal } \\
\text { Year }\end{array}$ & $\begin{array}{c}\text { Total Aid } \\
\text { Disbursement }\end{array}$ & $\begin{array}{c}\text { Swiss aid } \\
\text { Disbursement }\end{array}$ & $\begin{array}{c}\text { Disbursement as \% of } \\
\text { Commitment }\end{array}$ \\
$2001 / 02$ & 16457.1 & 846.77 & 5.15 \\
$2002 / 03$ & 16189 & 903.57 & 5.58 \\
$2003 / 04$ & 17523.9 & 932.72 & 5.32 \\
$2004 / 05$ & 18797.4 & 909 & 4.84 \\
$2005 / 06$ & 14384.8 & 1217.13 & 8.46 \\
$2006 / 07$ & 15885.5 & 1312.78 & 8.26 \\
$2007 / 08$ & 18942.4 & 1287.13 & 6.81 \\
$2008 / 09$ & 23657.3 & 1187.18 & 5.02 \\
$2009 / 10$ & 10126.10 & 2184.75 & 17.86 \\
$2010 / 11$ & 10797.10 & 2763.24 & 25.59 \\
$2011 / 12$ & 10452.97 & 3341.73 & 31.96 \\
$2012 / 13$ & 95995.12 & 4176.710 & 43.50 \\
$2013 / 14$ & 10366.48 & 3385.352 & 32.65 \\
$2014 / 15$ & 10207.55 & 3246.740 & 31.80 \\
Total & $\mathbf{2 8 9 7 8 2 . 7 2}$ & $\mathbf{2 7 6 9 4 . 8 0 2}$ & $\mathbf{2 3 2 . 8}$ \\
\hline
\end{tabular}

Source: Various issues of Economic Survey M OF/NG, SDC country program reports, SWC budget and annual reports and Foreign Aid division MOF/NG [4] and [5] and [13]

In 2001/02 the total aid disbursement was Rs.16457.1 million whereas the Swiss aid disbursement is only 846.77 million. Which is $5.15 \%$ in relation to the total aid disbursement? In FY 2005/06 total aid disbursement is increased in nature but Swiss aid disbursement is decreased in 2005/06 the total aid disbursement was 14384.8 million and Swiss aid disbursement was only 1217.13 million whereas the. In 2009/10 total aid was Rs. 10726.10 disbursement Swiss aid disbursement with disbursement $9 \%$ of communities was $17.66 \%$. The total aid disbursement is steady increasing in nature from FY 2009/10 
to 2014/15. Compared to previous year. The total aid disbursement was increased by more than year 2009/10 the Swiss aid disbursement was increasing in nature and again decreased in 2013/14to 2014/15 in relation to the total aid disbursement the Swiss aid occupied. The share from nearly $5 \%$ to nearly 43.5\%. In fiscal year 2009/10the Swiss aid again decreased and occupied $17.66 \%$ of total aid disbursement.

Table10 also exhibits that the Swiss aid disbursement during the study period is in fluctuating in nature. Swiss country program Repot 2008/09 clearly indicates low performance of aid utilization of Nepal government. So, they are not increased to increase the aid disbursement in Nepal. The SDC report also show the terrible internal war in Nepal so they are not interested to increase their project but want to increase humanitarian aid in Nepal

Table 11: Total Aid and Swiss Aid Commitment and Disbursement

(Rs. in million)

\begin{tabular}{|c|c|c|c|c|c|c|c|}
\hline $\begin{array}{l}\text { Fiscal } \\
\text { Year }\end{array}$ & $\begin{array}{l}\text { Total Aid } \\
\text { Commitment }\end{array}$ & $\begin{array}{l}\text { Total Aid } \\
\text { Disbursement }\end{array}$ & $\%$ & $\begin{array}{l}\text { Swiss aid } \\
\text { Commitment }\end{array}$ & $\begin{array}{l}\text { Swiss aid } \\
\text { Disbursement }\end{array}$ & $\%$ & Difference \\
\hline $2000 / 01$ & 39643 & 15031.9 & 37.92 & 484.94 & 849.94 & 75.03 & 37.11 \\
\hline $2001 / 02$ & 32022.1 & 16457.1 & 51.39 & 888.8 & 888.8 & 95.27 & 43.88 \\
\hline $2002 / 03$ & 1852.5 & 16189 & 88.21 & 996.5 & 996.5 & 90.67 & 2.46 \\
\hline 2003/04 & 20448 & 17523.9 & 85.70 & 996.82 & 996.82 & 93.57 & 7.87 \\
\hline $2004 / 05$ & 31287 & 18797.4 & 60.08 & 971.69 & 971.69 & 93.55 & 33.47 \\
\hline $2005 / 06$ & 33227.7 & 14384.8 & 43.29 & 1270.6 & 1270.6 & 95.79 & 52.50 \\
\hline $2006 / 07$ & 43202.7 & 15885.5 & 36.77 & 1292.35 & 1292.35 & 101.58 & 64.81 \\
\hline $2007 / 08$ & 23738 & 18942.4 & 79.67 & 1399 & 1399 & 92.00 & 12.33 \\
\hline 2008/09 & 38125.3 & 23657.3 & 62.01 & 1290.84 & 1290.84 & 91.97 & 29.96 \\
\hline $2009 / 10$ & 1884.66 & 12369.9 & 53.72 & 1300 & 1300 & 16.80 & 36.92 \\
\hline $2010 / 11$ & 1153.40 & 10797.1 & 936.11 & 3702.2 & 370.22 & 74.63 & 48.57 \\
\hline $2011 / 12$ & 7582.79 & 10453.0 & 13.78 & 1010 & 1010 & 33.08 & 19.3 \\
\hline $2012 / 13$ & 5507.3 & 9599.5 & 25.69 & 1080 & 1080 & 38.67 & 12.98 \\
\hline $2013 / 14$ & 2125.91 & 10366.5 & 48.76 & 2450 & 2450 & 13.81 & 34.95 \\
\hline $2014 / 15$ & 1195.56 & 10207.6 & 8.53 & 2500 & 2500 & 12.98 & 4.48 \\
\hline Total & 282995.92 & 220662.9 & 1631.63 & 21633.74 & 18666.76 & 1019.4 & 360.6 \\
\hline
\end{tabular}

Source: [4] and [5].

Table 11 exhibits the percentage difference between total aid commitment and disbursement and the Swiss aid commitment and disbursement. At the beginning of the study period the total foreign aid commitment was Rs. 320221 million and disbursement was Rs.16457.1 million which is only $51.39 \%$ in relation to aid commitment. At the same time Swiss aid commitment was Rs.888.8 million and disbursement was Rs. 846.77 million which is $95.27 \%$ in relation to the aid commitment. The percentage difference between them is $43.88 \%$. In FY 2002/03 the total foreign aid disbursement was $88.21 \%$ in relation to the commitment whereas the Swiss aid disbursement in relation to the commitment was $90.67 \%$. The percentage difference between them is $2.46 \%$ in FY 2003/04the total foreign aid disbursement in relation to commitment was $85.70 \%$ and the Swiss aid disbursement in relation to commitment was $93.70 \%$. The percentage difference between them is $7.87 \%$. At this period the total aid commitment was greater than commitment because of establishment of democratic government. Swiss aid disbursement is not poor which is more than 72\%. In FY 2008/09 total aid disbursement in relation to aid commitment was just $62 \%$ but the Swiss aid disbursement increased to external was $91.97 \%$. At the period the total Swiss aid disbursement was greater than committed amount. In FY 2009/10 the total aid disbursement was 53.72\% of the committed amount but the Swiss aid disbursement was $16.80 \%$ of aid commitment which is $36.92 \%$ greater in comparison to Swiss aid disbursement. In FY 2010/11 total aid disbursement in comparison to commitment was $26.06 \%$ and the Swiss aid disbursement in relation to commitment was $74.63 \%$ which is $48.57 \%$ more than the total aid 
commitment disbursement ratio from FY 2010/11 to 2014/15. The performance of Swiss aid disbursement as compared to total aid disbursement was poor. In FY 2011/12 total foreign aid disbursement was $13.78 \%$ of committed amount which is $19.3 \%$ greater in comparison to total aid disbursement. From FY 2012/13 onwards Swiss aid disbursement in relation to aid commitment were greater in comparison to total aid disbursement in relation to total aid commitment, i.e., $12.98 \%$ difference in FY 2012/13, 34.95\% in FY 2013/14, 4.48\% 2014/15. During the study period the total aid commitment is Rs. 396168.8 million and the amount of disbursement is Rs. 229073.9 million which is $57.82 \%$ of the committed amount. The Swiss aid commitment is Rs. 15115.93 million and disbursement is Rs. 14245.52 million which is $94.24 \%$ of the committed amount. The overall percentage difference between the total aid commitment disbursement and the Swiss aid commitment disbursement is $63.42 \%$. The existing condition of the Swiss aid disbursement in relation to total aid disbursement shows that Swiss aid disbursement ratio in relation commitment ratio were high in most years. It is due to project based investment, investment through user groups and NGOs. The Swiss investment is handing by SDC through projects in remote areas. The Swiss individual investment flows through SWC in selected NGOs and INGOs.

\subsection{Sectoral Commitment and Disbursement of Swiss Aid}

Over the period of last five decades the volume of the Swiss aid to Nepal has gradually increased and it covers all the major sectors of our economy. The analysis of Swiss assistance of the last 14 years indicates that the volume has steadily gone up and the areas of co-operation are in line with the priority as set by successive development plans.

Swiss government, INGO and individuals have made available assistance to almost all the sectors of our economy. Major benefited areas of Swiss assistance are agriculture, transport and communication, technical industry and education, social services and other sector including phase out program.

Table 12: Swiss Aid Commitment by Sectors

(Rs. In Million)

\begin{tabular}{|c|c|c|c|c|c|c|c|c|c|c|c|}
\hline $\begin{array}{l}\text { Fiscal } \\
\text { Year }\end{array}$ & Agriculture & $\%$ & Transport & $\%$ & Industry & $\%$ & $\begin{array}{l}\text { S. } \\
\text { Service }\end{array}$ & $\%$ & Other & $\%$ & Total \\
\hline $2000 / 01$ & 291.22 & 34.26 & 204.44 & 24.05 & 175.9 & 20.7 & 153.85 & 18.1 & 24.53 & 2.89 & 849.94 \\
\hline $2001 / 02$ & 153.79 & 17.30 & 332.33 & 37.39 & 187.05 & 21 & 110.41 & 12.4 & 105.2 & 11.84 & 888.8 \\
\hline $2002 / 03$ & 190.04 & 19.07 & 364.24 & 36.55 & 217.78 & 21.9 & 113.17 & 11.4 & 111.3 & 11.17 & 996.5 \\
\hline $2003 / 04$ & 200.34 & 20.10 & 371.09 & 37.23 & 173.74 & 17.4 & 153.83 & 15.4 & 97.82 & 9.81 & 996.82 \\
\hline $2004 / 05$ & 211.92 & 21.81 & 307.91 & 31.69 & 195.36 & 20.1 & 146.3 & 15.1 & 110.2 & 11.34 & 971.69 \\
\hline $2005 / 06$ & 350.08 & 27.55 & 369.95 & 29.12 & 260.83 & 20.5 & 126.6 & 9.96 & 163.1 & 12.84 & 1270.6 \\
\hline $2006 / 07$ & 333.18 & 25.78 & 380.72 & 29.46 & 268.42 & 20.8 & 146.13 & 11.3 & 163.9 & 12.68 & 1292.35 \\
\hline $2007 / 08$ & 374.5 & 26.77 & 420.58 & 30.06 & 284.68 & 20.3 & 127.55 & 9.12 & 191.7 & 13.70 & 1399 \\
\hline $2008 / 09$ & 445.45 & 34.51 & 264.97 & 20.53 & 0 & 0 & 493.57 & 38.2 & 86.85 & 6.73 & 1290.84 \\
\hline $2009 / 10$ & 251.6 & 25.49 & 328.72 & 20.38 & 218.64 & 17.46 & 163.83 & 13.12 & 106.14 & 10.29 & 1068.93 \\
\hline $2010 / 11$ & 264.3 & 27.73 & 417.31 & 30.18 & 212.63 & 16.33 & 253.17 & 16.74 & 78.14 & 7.84 & 1225.55 \\
\hline $2011 / 12$ & 215.7 & 22.15 & 371.81 & 23.14 & 179.42 & 15.64 & 298.16 & 19.24 & 116.2 & 10.53 & 1181.29 \\
\hline
\end{tabular}




\begin{tabular}{|l|l|l|l|l|l|l|l|l|l|l|l|}
\hline $2012 / 13$ & 261.9 & 26.18 & 335.71 & 21.15 & 257.46 & 18.62 & 412.16 & 29.31 & 96.4 & 8.34 & 1363.63 \\
\hline $2013 / 14$ & 421.3 & 31.37 & 337.31 & 22.15 & 216.14 & 19.24 & 418.17 & 28.24 & 104.3 & 9.84 & 1497.22 \\
\hline $2014 / 15$ & 446.1 & 33.19 & 419.12 & 31.28 & 219.15 & 16.70 & 464.18 & 31.82 & 182.3 & 12.31 & 1730.85 \\
\hline Total & $\mathbf{4 4 1 1 . 4 2}$ & $\mathbf{3 9 3 . 2 6}$ & $\mathbf{5 2 2 6 . 2 1}$ & $\mathbf{4 2 4 . 3 6}$ & $\mathbf{3 0 6 7 . 2}$ & $\mathbf{2 6 6 . 6 9}$ & $\mathbf{3 5 8 1 . 1}$ & $\mathbf{2 7 9 . 4 5}$ & $\mathbf{1 7 3 8 . 1}$ & $\mathbf{1 5 2 . 1 5}$ & $\mathbf{1 8 0 2 4}$ \\
\hline
\end{tabular}

Source: 1. various issues of SDC country program reports, SWC budget and annual reports and Foreign Aid division MOF/NG [4]and

[5].

Justification:

1. Agricultural irrigation and forestry (Natural Resource management)

2. Transport power and communication

3. Small industry commerce and Technical education

4. Social services including humanitarian's aid

5. Other sector including consolidated and phased out program

Expenditure

The table 12 reflects the Swiss aid commitment in different sector during the study period. The Swiss aid has concern with debt relief measures. Transport, agriculture industry and technical education, social services sectors including humanitarian aid and other sectors i.e., consolidate or phased out program. The maximum amount of aid commitment is transport power and communication which occupied Rs.5226.31 million which is $30 \%$ of the total aid commitment. The second priority has been given in agriculture irrigation and in the FY 2001 to 2002 the percentage occupied by agriculture. Sector is $17.30 \%, 19.07 \%$, 2010 of $012.81 \% 27.55 \%, 25.76 \%, 26.77 \% 34.51 \%, 25.49 \%, 27.73 \%, 22.15 \%$, $26.18 \%, 31.37 \%$ and $23.10 \%$. The percentage occupied by transport year is $24.05 \%, 37.39 \%, 36.5 \%$, $37.23 \%, 31.62 \%, 29.12 \%, 29.46 \%, 30.06 \%, 20.53 \%, 20.38 \%, 30.18 \%, 23.14 \%, 21.15 \%, 22.15 \%$ and $31.28 \%$.

The percentage occupied by industry sector from FY $2001 / 2002$ to $2014 / 15$ is $20.70 \%, 21 \%, 21.9 \%$, $17.4 \%, 20.1 \%, 20.5 \%, 20.8 \%, 20.30 \%$, of $17.465 \%, 16.33 \%, 15.64 \%, 18.62 \%, 19.24 \%$ and $16.70 \%$. And the percentage occupied by the social services sector from FY 2001/2002 to 2014/15 is $18.1 \%$, $12.4 \%, 11.4 \%, 15.4 \%, 15.1 \%, 9.96 \%, 29.31 \%, 24.24 \%$ and $31.82 \%$.

Forestry sector which is known a natural resources management in Swiss language. In this sector total aid commitment during the study period has Rs. 4411.42 million which is $26 \%$ of the total aid commitment. The third priority has been given in social service. This sector occupies $18 \%$ of the total aid commitment. In these sector service sector. In these sector 3.67.2 million grants has been committed. Least priority has given in other sector. In this sector Rs.1738.1, million aids have been committed. In year wise comparison in the beginning of the fiscal year agriculture was given topmost priority in aid commitment. The total Swiss aid commitment in agriculture was Rs. 4411.42 million with of occupied. The second priority was given in the Transport. The total aid commitment in this RS. 5226.21 of the total committed amounts. The total aid commitment in industry sector is Rs. 3067.2 million social service of Rs. 3181.1 and other sector is RS. 17138.1 million Commitment. In FY 2001/002 agriculture sector was given lest priority in aid commitment. This occupied only $17.30 \%$ share of total aid commitment. In that year Swiss individuals and NGOs provided agricultural aid but Swiss government doesn't provided aid in this sector. Transport was given priority. In this sector Rs. 332.33 million aids were committed which is $37.69 \%$ of total aid commitment. Second priority in aid commitment was given in transport sector which was Rs.187.05 million and $21 \%$ of the total aid commitment. At that period no aid was committed in industry, commerce and technical education occupied $36.55 \%$, small industry commerce and technical education occupied $21.90 \%$ and social service sector occupied $11.4 \%$ and other sectors occupied $11.70 \%$. In this year agriculture sector was given topmost priority of all. In FY 2002/03the total aid commitment was Rs.1068.92 million which was decreased by Rs. 221.91 million as compared to the previous year. In this year the agriculture sector was given top priority. This sector occupied $34.51 \%$ share of the total aid commitment. Second priority was given in service sector. This sector occupied $38.2 \%$ of the total aid commitment. Other service sector was given the third priority and occupied $6.73 \%$. No aid commitment was made in small industry, commerce and technical 
education sector. In FY 2014/15 first priority was given in agriculture sector. Second priority was in transport, third in s. service and fourth in transport sector. In this year industry sectors were given least priority. In this year the total aid commitment was Rs. 1730.85 million. In FY 2014/15 the total Swiss aid commitment was increased by Rs.233.63 million as compared to the previous year and became Rs.846.62 million. In the year agriculture sector was given topmost priority in aid commitment. 33.19\%, share is occupied by this sector. In FY 2008/09 the total aid commitment was decreased as compared to the previous year by Rs. $108.16 \%$ million. in this year social service sector occupied $38.24 \%$ and agriculture sector $34.51 \%$, transport sector occupied $2035 \%$ and other sectors occupies $6.72 \%$. In this year there is no aid commitment in industry sector. The trend of commitment clearly shows that like other donors, the Swiss government and individuals are interested to develop infrastructure of nation. During the study period the total Swiss aid disbursement is Rs 14245.52 million which is more than 94 percent of the committed amount. The total Swiss aid disbursement during study period in relation to total foreign aid commitment is only 6.22 percent. Table 13 shows the sectoral disbursement of Swiss aid during the study period.

Table 13: Swiss aid Disbursement by Sectors

(Rs. In Million)

\begin{tabular}{|c|c|c|c|c|c|c|c|c|c|c|c|}
\hline $\begin{array}{l}\text { Fiscal } \\
\text { Year }\end{array}$ & Agriculture & $\%$ & Transport & $\%$ & Industry & $\%$ & $\begin{array}{l}\text { S. } \\
\text { Service }\end{array}$ & $\%$ & Other & $\%$ & Total \\
\hline $2000 / 01$ & 79.46 & 12.46 & 282.34 & 44.28 & 136.07 & 21.34 & 63.53 & 9.96 & 76.27 & 11.96 & 637 \\
\hline $2001 / 02$ & 108.44 & 12.81 & 262.62 & 31.01 & 200 & 23.62 & 77.74 & 9.18 & 19.8 & 23.38 & 846 \\
\hline $2002 / 03$ & 110.38 & 12.22 & 363.07 & 40.18 & 156.13 & 17.28 & 117.98 & 13.06 & 156 & 17.27 & 903 \\
\hline $2003 / 04$ & 223.78 & 23.99 & 187.28 & 20.08 & 128.89 & 13.82 & 281.31 & 30.16 & 111.5 & 11.95 & 932 \\
\hline $2004 / 05$ & 212.13 & 23.34 & 312.33 & 34.36 & 83.38 & 9.17 & 247.64 & 27.24 & 53.52 & 5.89 & 90 \\
\hline $2005 / 06$ & 268.13 & 22.03 & 240.12 & 19.73 & 97.93 & 8.05 & 518.69 & 42.62 & 92.26 & 7.58 & 1217 \\
\hline $2006 / 07$ & 290.61 & 22.14 & 366.53 & 27.92 & 102.12 & 7.78 & 441.65 & 33.64 & 111.9 & 8.52 & 1312 \\
\hline $2007 / 08$ & 314.49 & 24.43 & 416.39 & 32.35 & 0 & 0 & 447.54 & 34.77 & 108.7 & 8.45 & 1287 \\
\hline $2008 / 09$ & 314.42 & 26.48 & 277.08 & 23.34 & 0 & 0 & 475.03 & 40.01 & 120.7 & 10.16 & 1187 \\
\hline $2009 / 10$ & 315.42 & 27.48 & 356.53 & 26.53 & 109 & 9.91 & 317.4 & 22.15 & 101 & 7.12 & 1199 \\
\hline $2010 / 11$ & 298.61 & 25.14 & 367.66 & 41.08 & 173 & 22.61 & 418.12 & 29.14 & 121 & 8.17 & 1378 \\
\hline $2011 / 12$ & 317.42 & 27.48 & 416.40 & 30.88 & 181 & 24.71 & 440.13 & 33.19 & 151 & 10.12 & 1505 \\
\hline $2012 / 13$ & 325.13 & 29.78 & 330.33 & 24.18 & 156 & 20.14 & 451.13 & 38.17 & 120 & 11.13 & 1382 \\
\hline $2013 / 14$ & 335.42 & 33.18 & 417.18 & 31.82 & 173 & 24.11 & 471.21 & 39.18 & 137 & 15.18 & 1533 \\
\hline $2014 / 15$ & 340.49 & 36.17 & 419.22 & 33.79 & 191 & 26.44 & 182.22 & 41.01 & 139 & 18.19 & 1271 \\
\hline Total & 3854.33 & 359.13 & 5015.08 & 461.53 & 1887.52 & 228.98 & 4951.3 & 443.48 & 1619.7 & 175.07 & 16679 \\
\hline
\end{tabular}

Source: various issues of SDC country program reports, SWC budget and annual reports and Foreign Aid division MOF/NG [4]and [5]. 
Table 13 clearly indicates that transport power and communication sector has been given the first priority in aid disbursement. During the study period Rs. 5015.08 million Swiss aids have been disbursed in this sector which is $31 \%$ of the total Swiss aid disbursement. Third priority has been given agricultural irrigation and forestry sector. In this sector Rs.3854.33 million aids have been disbursed which is $22 \%$ of the total Swiss aid disbursement. Second priority has been given to social service sector. In this sector Rs. 4951.3 million Swiss aid has been disbursed which is $21 \%$ of the total aid disbursement. Least priority has been given to industry commerce and technical education sector and other sector.

In year wise comparison, the first two years i.e., 2001/02 and 2002/03 Rs. 1084.44 and 110.38 million was disbursed in the agricultural sector. In this sector $12.81 \%$ Swiss aid was disbursed in FY 2001/02 and $12.21 \%$ in FY 2002/03. In transport power and communication. Sector $31.01 \%$ Swiss aid was industry in FY 2001/02 commerce and technical education. In this sector 40.18\% FY 2002/03. Least priority has been given in social service sector. Only $0.20 \%$ aid was disbursed in FY 2002/03 and $13.06 \%$ in FY 2002/03. In these two years other sectors occupied $23.38 \%$ and $17.27 \%$ respectively. In agriculture sector the $\%$ of share was decreased by $059 \%$. Transport sector was increased by $9.17 \%$. Industry sector was decreased by $6.37 \%$, social service was increased by $3.88 \%$ and other sectors were decreased by $6.11 \%$. In totality the aid disbursement was Rs. 6.37 million. Other sectors were given the priority and occupied $17.27 \%$ which is $6.11 \%$ than the previous year disbursement in percentage. Transport sector $40.18 \%$ aid was disbursed which is $9.17 \%$ greater than the previous year. In FY 2003/04agriculture and s. service sectors were given topmost priority in aid disbursement. In this year the total aid disbursement was Rs. 932 million. In total the aid disbursement was increased Rs. 29 million which is $3.21 \%$ in comparison to previous year. Transport and other sector occupied $20.08 \%$ and $11.95 \%$ respectively. In transport sector the aid was decreased in mount and decreased in percentage share by $20.1 \%$. Other sectors exhibit the same result. The aid was decreased by Rs. 44.5 million in amount and decreased $5.32 \%$ in comparison in its share. The industry sector occupied $13.82 \%$ share. In this sector the amount of aid disbursement was increased by Rs. 19 million but percentage share was decreased by $3.46 \%$. Third priority was given in industry sector. In this sector $14.74 \%$ aid was disbursed which is greater by Rs. 128.89 million but percentage share was decreased by $3.46 \%$. Social service sector occupied only $30.16 \%$. In this sector $17.1 \%$ aid has increased as compared to the previous year.

The third priority was figure to the agriculture sector. The aid disbursement is Rs. 317.42 million was Rs. 18.81 million greater than previous years, with the percentage occupied by $27.48 \%$ which is $2.34 \%$ sector than previous years.

In FY 2012/13, the total aid disbursement was Rs. 1382 which is Rs. 123 million was more than previous years with most primarily sign to the s. service sector. Transport and agriculture the sector, with the aid disbursement of Rs. 451.13 million, 33.33 million and 325.13 million with the percentage than occupied with $38.17 \%, 24.16 \%$ and $29.78 \%$. It is percentage there increased by $4.98 \%$ in service sector, $6.7 \%$ decreased in Transport and $2.3 \%$ increase in agriculture sector, service.

In FY 2013/14 the total aid disbursement was Rs.1533 million is Rs.151 million greater than previous years with most percentage to the service sector, Transport sector and agriculture sector with the aid disbursement by Rs. 471.21 million, 417.18 million and 335.42 million with the percentage there occupied with $39.18 \%, 31.82 \%$ and $33.18 \%$. The percentage there increased by $1.01 \%, 7.64 \%$ and $3.4 \%$ in and service, Transport and agriculture sector percentage.

In FY 2014/15 the total aid disbursement was Rs. 1271 million which Rs. 262 les then previous years with must previous to the transport sector agriculture with the aid disbursement of Rs. 119.22 million and 340.49 million with the percentage there occupied with $33.79 \%$ and $36.17 \%$. The percentage service increased by $1.97 \%$ and $2.99 \%$ in transport and agriculture the service percentage

In FY 2009/10 the total aid disbursement was Rs. 1199 million which was Rs. 17 million sector then the previous year. In this year's Transport sector was service transport priority and $26.53 \%$. Then occupied by this sector with Rs. 356.69 million aid disbursement this sector. Then second priority was given the social service sector. In this sector Rs. 317.4 million aid were disbursed which is 157.6 million less than previous year. The percentage there were decreased by $17.86 \%$. Then third priority was service to the agriculture sector. Disbursing Rs. 315.42 million with percentage occupied by $27.48 \%$ which increasing by $10 \%$. 
In FY 2010/11 the total aid disbursement was Rs. 1378 million which was Rs. 179 million less than previous years. In this year's. Service sector was given most priority and $29.14 \%$ there was occupied by this sector with Rs. 418.12 million aid disbursed in this sector. Second primarily was given most priority and $29.14 \%$ share were occupied by this sector with Rs.418.12 million aid was disbursed which is 11.13 million greater than previous year. The percentage share increased by $14.55 \%$. The third priority was given to the agricultural sector, disbursing Rs.298.61 million with percentage occupied by $25.14 \%$ which the decreased Rs. $2.34 \%$.

In FY 2011/12 the total aid disbursement was Rs. 105 million which was RS. 127 million sector than previous years. In this years' service sector was given most priority and $33.19 \%$ of these was occupied by this sector with Rs. 440.13 million aid disbursed in this sector. Secondary serious was given to the transport sector with $416.40 \%$ million aid disbursement which is 48.74 million sector than primes in years with percentage occupied by 30.88 , which is this less $10.2 \%$ then previous years.

\section{CONCLUSION :}

The growth of foreign aid in Nepal during the study period is taking a rapid tempo but economic development of Nepal in comparison aid receiving is found very low due to some specific cause's, i.e., low absorptive capacity due to low performance of aid management, visionless politicians in aid management, political insatiability, rampant corruption aid utilizing projects, and the trend of grabanything from external sources. But the Swiss aid in Nepalese economy is found very effective due to some special features, i.e., working in remote areas, keeping on track, strong rural affiliation, preference given for environmental protection, project-based investment, following bottom up approach in development, $100 \%$ grant amount and implementation of labor intensive technique, the Swiss aid directly affects the poverty reduction of Nepal. In amount Swiss aid is much less but is efficiency is widespread in increasing people's living standard. Due to $100 \%$ grant Swiss aid doesn't create fiscal burden in the economy. Thus, it is necessary to increase Swiss aid and Swiss project in Nepal for sustained and broad based economic development.

\section{REFERENCES :}

[1] Elikand, B. M. \& Mapunjo, J. K. G. (2004). An independent review of Poverty Reduction and aid Effectiveness. The Reality of Aid Journal. Manila: Zed Books London and IBON Books.

[2] Gervase, M., Glora, S. \& Timothy, J. (1997). Effective Aid Management, the Case of Botswana. Foreign Aid in Africa: Africa: Learning Country Experiences.

[3] Ministry of Finance (1990/091) to 2014/15), Economic Survey, Kathmandu: MOF assessed from www.aims.mof.gov.np

[4] Ministry of Finance (2019). Foreign Aid Policy of Nepal 2019 Kathmandu: MOF assessed from www.mof.gov.np

[5] National Plannig Commission (First to thirteenth Plan). Kathmandu: NRB from www.nrb.org.np and www.npc.gov.np

[6] Organization for Economic Co-operation and Development (1991). Development Cooperation Report. Geneva: OECD. https://data.oecd.org/

[7] Social Welfare Council (1995/96 to 2004/05), Budget Report. Kathmandu: SWC. https://www.swc.org.np/

[8] Swiss Agency for Development cooperation (1993). Country Program For Nepal1993-94. Ekantakuna:

SADC.https://www.eda.admin.ch/countries/nepal/en/home/representations/embassykathmandu/embassy-tasks/cooperation-office.html

[9] Swiss Agency for Development Corporation (1997). Country Program for Nepal 1998-2004. Ekantakuna:SADC. $\quad$ https://www.eda.admin.ch/countries/nepal/en/home/representations/ embassy-kathmandu/embassy-tasks/cooperation-office.html

[10] Swiss Agency for Development Corporation (1998). Country Program for Nepal 1998-2004. Ekantakuna:

SADC. 
https://www.eda.admin.ch/countries/nepal/en/home/representations/embassykathmandu/embassy-tasks/cooperation-office.html

[11] Swiss Agency for Development Corporation (2005). Country Program for Nepal 1998-2004. Ekantakuna: SADC. https://www.eda.admin.ch/countries/nepal/en/home/representations/ embassy-kathmandu/embassy-tasks/cooperation-office.html

[12] UNDP (2005). Human Development Report. Pulchowk: UNDP. http://hdr.undp.org/en/content/human-development-report-2005

[13] WB (2005). World Development Report. Kathmandu: WB. https://documents.worldbank.org/en/publication/documentsreports/documentdetail/554071468182337250/world-development-report-2005-a-betterinvestment-climate-for-everyone 